\title{
$\Delta$ Np63-driven recruitment of myeloid-derived suppressor cells promotes metastasis in triple-negative breast cancer
}

\author{
Sushil Kumar, ${ }^{1}$ David W. Wilkes, ${ }^{1}$ Nina Samuel, ${ }^{1}$ Mario Andres Blanco, ${ }^{1}$ Anupma Nayak, ${ }^{2}$ Kevin Alicea-Torres, ${ }^{3}$ Christian Gluck, ${ }^{4}$ \\ Satrajit Sinha, ${ }^{4}$ Dmitry Gabrilovich, ${ }^{3}$ and Rumela Chakrabarti' \\ 'Department of Biomedical Sciences, School of Veterinary Medicine, University of Pennsylvania, Philadelphia, Pennsylvania, USA. ²Department of Pathology and Laboratory Medicine at the Hospital of the \\ University of Pennsylvania, University of Pennsylvania Perelman School of Medicine, Philadelphia, Pennsylvania, USA. ${ }^{3}$ The Wistar Institute, Philadelphia, Pennsylvania, USA. ${ }^{4}$ Department of Biochemistry, \\ State University of New York, Buffalo, New York, USA.
}

\begin{abstract}
Triple-negative breast cancer (TNBC) is particularly aggressive, with enhanced incidence of tumor relapse, resistance to chemotherapy, and metastases. As the mechanistic basis for this aggressive phenotype is unclear, treatment options are limited. Here, we showed an increased population of myeloid-derived immunosuppressor cells (MDSCs) in TNBC patients compared with non-TNBC patients. We found that high levels of the transcription factor $\Delta$ Np63 correlate with an increased number of MDSCs in basal TNBC patients, and that $\triangle \mathrm{Np63}$ promotes tumor growth, progression, and metastasis in human and mouse TNBC cells. Furthermore, we showed that MDSC recruitment to the primary tumor and metastatic sites occurs via direct $\triangle$ Np63-dependent activation of the chemokines CXCL2 and CCL22. CXCR2/CCR4 inhibitors reduced MDSC recruitment, angiogenesis, and metastasis, highlighting a novel treatment option for this subset of TNBC patients. Finally, we found that MDSCs secrete prometastatic factors such as MMP9 and chitinase 3-like 1 to promote TNBC cancer stem cell function, thereby identifying a nonimmunologic role for MDSCs in promoting TNBC progression. These findings identify a unique crosstalk between $\triangle \mathrm{Np63}^{+}$TNBC cells and MDSCs that promotes tumor progression and metastasis, which could be exploited in future combined immunotherapy/chemotherapy strategies for TNBC patients.
\end{abstract}

\section{Introduction}

Breast cancer is a devastating disease that, despite significantly advanced treatment options, claims around 50 million lives per year worldwide. Breast cancer is classified into subtypes based on the expression of estrogen receptor (ER), progesterone receptor (PR), and human epidermal growth factor receptor 2 (HER2) (1). Triple-negative breast cancers (TNBCs) that are ER-PR HER2 account for about $20 \%$ of all breast cancers, and their lack of receptors renders them insensitive to endocrine-targeted therapy (2). Despite an initial response to systemic chemotherapy, patients with TNBC follow an aggressive course of progression and metastasis $(2,3)$. Tumor relapse, resistance to chemotherapy, and metastases are more prevalent in TNBC compared with other subtypes of breast cancer $(3,4)$, all contributing to the poor prognosis of TNBC patients. In spite of several ongoing studies, the mechanistic basis for the aggressive phenotype and higher metastatic potential of TNBCs remains unclear.

The progression and metastasis of solid tumors are influenced by the tumor microenvironment (TME), which is composed of tumor cells and other protumor stromal cells (5-7). Myeloidderived immunosuppressor cells (MDSCs) are a major component of the TME, and their increased number in blood is associated

Conflict of interest: The authors have declared that no conflict of interest exists Submitted: January 5, 2018; Accepted: August 28, 2018

License: Copyright 2018, American Society for Clinical Investigation.

Reference information: / Clin Invest. 2018;128(11):5095-5109.

https://doi.org/10.1172/JCI99673. with poor patient survival $(8,9)$. In mice, MDSCs are a poorly differentiated and heterogeneous $\mathrm{CD}_{11 \mathrm{~B}}{ }^{+} \mathrm{Gr} 1^{+}$population that can be further divided into 2 subsets: polymorphonuclear (PMN-MDSCs; Ly $6 \mathrm{G}^{+}$) and monocytic (M-MDSCs; Ly6C $\mathrm{C}^{+}$(9-11). In humans, PMN-MDSCs are defined by immature myeloid markers: PMN-MDSCs are CD14 ${ }^{-} \mathrm{CD} 11 \mathrm{~B}^{+} \mathrm{CD} 33^{+} \mathrm{CD} 15^{+} \mathrm{LOX}^{-1}{ }^{+}$and $\mathrm{CD} 66 \mathrm{~B}^{+}$ cells, and M-MDSCs are CD14 ${ }^{+} \mathrm{HLA}^{-\mathrm{DR}^{-/ 10}}$ cells (9). MDSCs promote tumor cell survival, angiogenesis, invasion into healthy tissues, and metastases $(8,9)$. MDSCs are produced in the bone marrow of tumor-bearing hosts and, in response to tumor-derived chemokines and hypoxia-induced factors, migrate to the primary tumor and metastatic niches to support metastases. While MDSCs in breast cancer have been evaluated in mouse models and circulating blood of patients, the distribution, number, and function of MDSCs in the TME of different subtypes of breast cancer are completely unknown. As overrepresentation of MDSCs in aggressive cancers could potentially contribute to their metastatic nature, we set out to determine whether MDSCs increase dissemination and promote colonization of the premetastatic niche to promote metastasis and recurrence of TNBCs.

p63, a transcription factor of the p53/p63/p73 gene family, is transcribed from 2 different promoters, resulting in 2 isoforms: one that possesses a full-length transactivation domain analogous to that of $\mathrm{p} 53$ (the TA isoform), and one that possesses a distant activation domain (the $\Delta \mathrm{N}$ isoform) (12-15). While TAp63 is considered a tumor suppressor (16), $\Delta \mathrm{Np} 63$ exhibits a predominantly oncogenic function in a number of cancers, including head and neck squamous cell carcinoma, in which it is primarily studied 
(17-21). Our earlier studies showed that $\Delta$ Np63 maintains normal mammary stem cell activity, and its upregulation promotes TNBC/basal breast cancer initiation by regulating Wnt signaling (21). Additional recent reports corroborated the oncogenic function of $\Delta \mathrm{Np} 63$ in breast cancer via the Sonic hedgehog and Notch pathways $(22,23)$. However, it remains unclear whether the oncogenic effects of $\Delta \mathrm{Np} 63$ act primarily in a tumor cell-autonomous manner or whether $\Delta \mathrm{Np} 63$ also dictates changes in the TME that may promote the aggressive nature of TNBCs.

In this study, we observed that TNBCs express higher levels of $\triangle N$ p63 and have a greater number of PMN-MDSCs in the TME compared with non-TNBCs in both clinical samples and human breast cancer xenografts. Additionally, we found that $\triangle$ Np63 promotes metastasis and is responsible for CXCL2- and CCL22-dependent recruitment of MDSCs to the primary tumor and metastatic sites. Furthermore, blocking this signaling axis with chemokine receptor inhibitors decreases angiogenesis and metastatic events significantly. More surprisingly, treatment of MDSCs with chemokines (CXCL2/CCL22) induces their secretion of protumorigenic factors such as chitinase 3-like 1 (CHI3L1) and MMP9, which enhance the tumor growth and metastatic potential of TNBCs. Together, our study identifies a novel non-

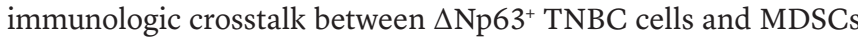
that promotes invasion, angiogenesis, and metastasis in the basal subset of TNBCs and that may be exploited for future combination therapy to prevent MDSC expansion or recruitment.

\section{Results}

Greater MDSC infiltration correlates with increased $\triangle N p 63$ expression in TNBC patients. The number and function of MDSCs in the TME of breast cancer patients remain unknown, and it has yet to be determined whether overrepresentation of MDSCs in TNBCs may directly contribute to their aggressive phenotype. To determine whether MDSCs are enriched in TNBC patient samples, we first used a CD11B antibody that marks myeloid cells. $\mathrm{H}$-score and abundance data based on immunohistochemistry (IHC) indicated that human TNBC (ER-PR-HER2-) breast cancer samples have higher $\mathrm{CD}_{11 \mathrm{~B}^{+}}$myeloid infiltrates than non-TNBC samples $\left(\mathrm{ER}^{+} \mathrm{PR}^{+} \mathrm{HER} 2^{-/+}\right)(P=0.001)$ (Supplemental Figure 1, A and B; supplemental material available online with this article; https://doi.org/10.1172/JCI99673DS1). These infiltrating myeloid cells were confirmed to be MDSCs in immunofluorescence assays using a combination of CD33 and S100A9 antibodies (24) $(P=0.002)$ (Figure 1A). Human MDSCs are

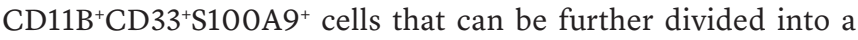
granulocytic subtype (PMN-MDSCs, also known as G-MDSCs) that coexpresses CD15 and LOX-1 (25) and a monocytic subtype (M-MDSCs). As we found an increased number of dual-positive CD15 ${ }^{+} \mathrm{LOX}-1^{+}$cells $(25)$ in TNBC compared with non-TNBC $(P=$ 0.001) patients (Figure 1B), our data suggest that TNBC patient tumors have more PMN-MDSCs than do non-TNBC patients.

The transcription factor $\triangle \mathrm{Np} 63$ is the predominant isoform of p63 expressed in breast cancer and is overexpressed in TNBCs compared with non-TNBCs $(21,26,27)$. Although $\triangle \mathrm{Np} 63$ contributes to tumor initiation by regulating tumor-initiating cells or cancer stem cells (21), its potential effects on TME-driven tumor progression have not yet been assessed. Therefore, we next determined whether
MDSC infiltration was related to $\Delta \mathrm{Np} 63$ expression in breast cancer patient samples. Notably, we found that $\Delta$ Np63 is overexpressed in the basal subset of TNBCs compared with non-TNBCs, and that $\triangle$ Np63-enriched TNBCs express higher levels of the $\Delta$ Np63-target gene K14 (Figure 1C and Supplemental Figure 1, C-E). Increased K14 expression is associated with the basal subtype of TNBC (Supplemental Figure 1D), which has a greater mitotic index and is more aggressive than other subtypes $(4,28,29)$.

Further analyses revealed a positive correlation between $\Delta$ Np63 and CD11B positivity $(r=0.49, P=0.0001), \Delta \mathrm{Np} 63$ and MDSC $\left(\mathrm{CD}_{3}{ }^{+}\right.$S100A9 $\left.{ }^{+}\right)$positivity $(r=0.54, P=0.01)$, and $\triangle \mathrm{Np} 63$ and PMN-MDSC $\left(\mathrm{CD} 15^{+} \mathrm{LOX}-1^{+}\right)$positivity $(r=0.34, P=$ 0.03) in TNBC tumors (Supplemental Figure $1 \mathrm{~F}$ and Figure 1, D and $\mathrm{E})$. In addition, we found a correlation $(r=0.62, P=0.08)$ between $\triangle \mathrm{Np} 63$ and K14 positivity in TNBC patient tumors, when TNBC patient tumors were stratified into $\triangle$ Np63-high- and $\Delta$ Np63-low-expressing tumors (Supplemental Figure 1G). Complementary assessment of $\triangle \mathrm{Np} 63$ expression in TNBC patients stratified into K14-high and -low cohorts revealed a strong trend $(P=0.06)$ toward higher $\Delta$ Np63 expression by K14-high tumors (Supplemental Figure 1H). These data indicate that basal-like TNBCs have a higher tendency to express more $\Delta$ Np63. In contrast, we did not observe any correlation between $\triangle \mathrm{Np} 63$ status and MDSC status $(r=0.021, P=0.927)$, PMN-MDSC status $(r=0.164, P=0.404)$, or K14 status $(r=0.005, P=0.98)$ in nonTNBC tumors (data not shown).

In complementary studies, we used human breast cancer cell xenografts from 2 human basal TNBC cell lines (HCC1806 and SUM159) and 1 human non-TNBC luminal cell line (MCF7). Similarly to human breast tumors, we found that TNBC xenografts

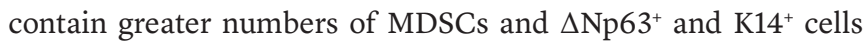
compared with non-TNBC xenografts (Supplemental Figure 2, A-C). Finally, we found that high levels of TP63 significantly correlate with reduced distant metastasis-free survival (DMFS) in the ER $^{-} \mathrm{PR}^{-}$and TNBC (ER-PR-HER2-) clinical breast cancer samples in the Kaplan-Meier Plotter (KM Plotter) database (ref. 30 and Figure 1, F and G). In contrast, we observed an inverse correlation between TP63 and DMFS in non-TNBC $\left(\mathrm{ER}^{+} \mathrm{PR}^{+} \mathrm{HER} 2^{+}\right)$patients (Figure 1H) in the KM Plotter database. Similar trends were observed in an independent data set (the METABRIC study; refs. 31, 32), in which high TP63 expression correlates with worsened prognosis in $\mathrm{ER}^{-}$and $\mathrm{PR}^{-}$patients (Supplemental Figure 2, D and E). Together, our data suggest that $\triangle \mathrm{Np} 63^{+}$cells and MDSC infiltrates are higher in the basal subset of TNBCs, and may be responsible for their greater incidence of metastasis.

Primary tumor growth, progression, and metastasis are dependent on $\triangle N p 63$ in human TNBC cells. To test the functional role of $\triangle \mathrm{Np} 63$ in TNBC tumor growth, progression, and metastasis, we performed shRNA-mediated $\triangle \mathrm{Np} 63$ knockdown studies in HCC1806 and SUM159 TNBC cell lines, the former of which expresses both red fluorescent protein (RFP) and Renilla luciferase to facilitate in vivo tracking (33). Isoform-specific knockdown (KD) of $\triangle \mathrm{Np} 63$ was confirmed at both the mRNA and the protein level in HCC1806 (Figure 2A and Supplemental Figure 3A) using published isoform-specific shRNA (21). Knockdown of $\triangle \mathrm{Np63}$ had no effect on TAp63 in these cells (Supplemental Figure 3A). Mammary fat pad injection revealed a decrease in primary tumor 
A

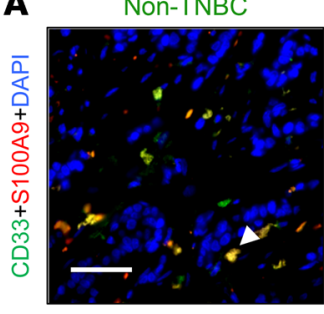

C

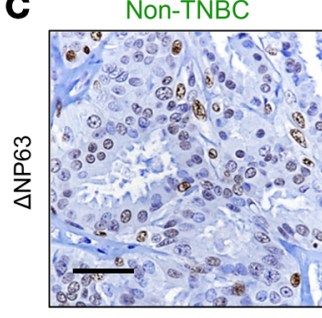

TNBC

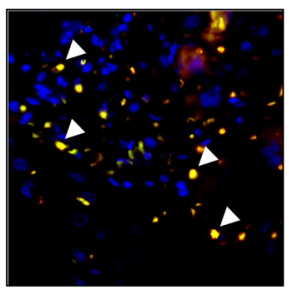

TNBC

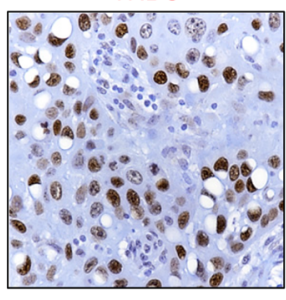

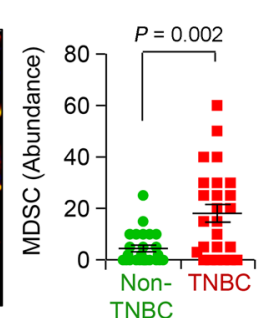
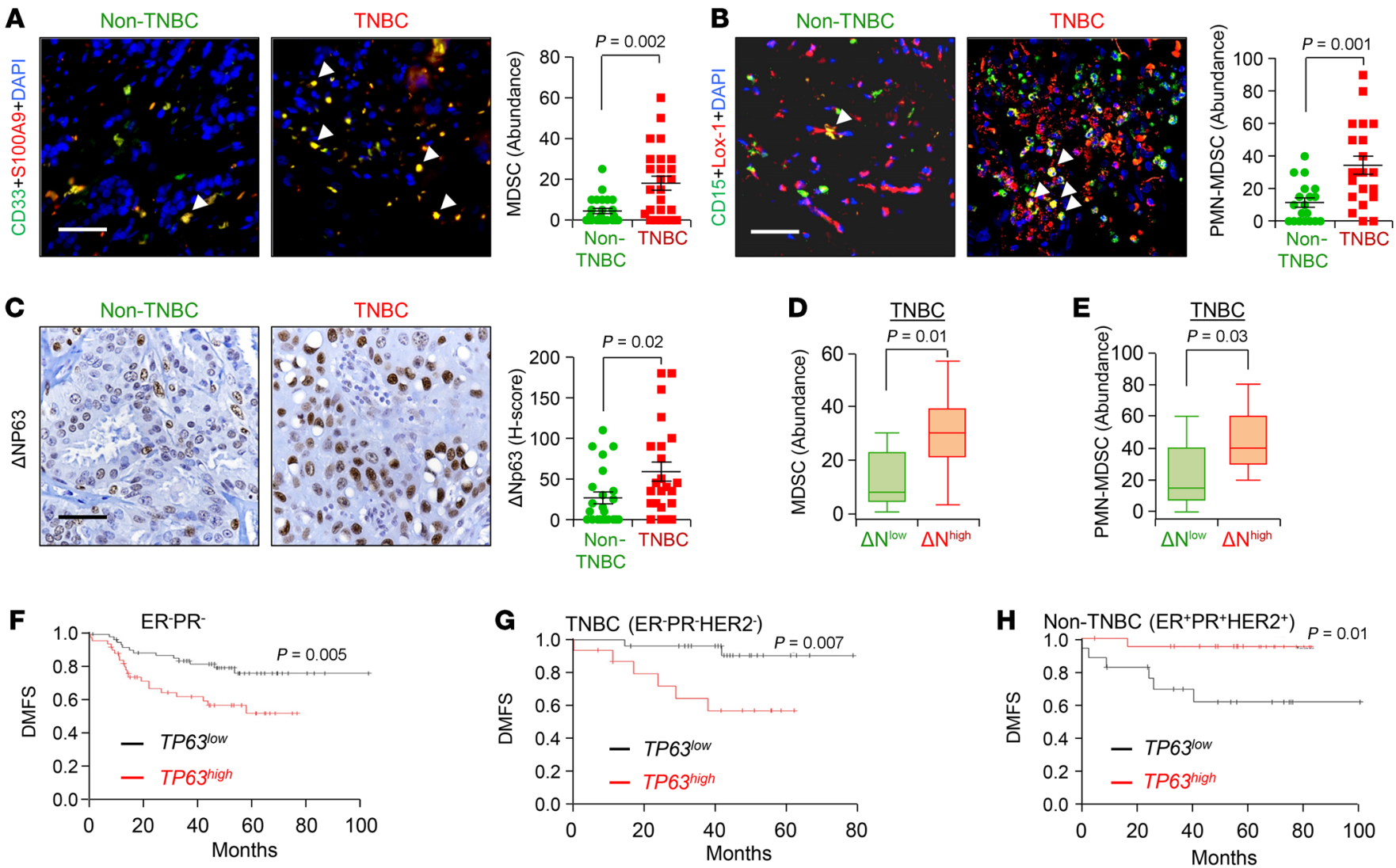
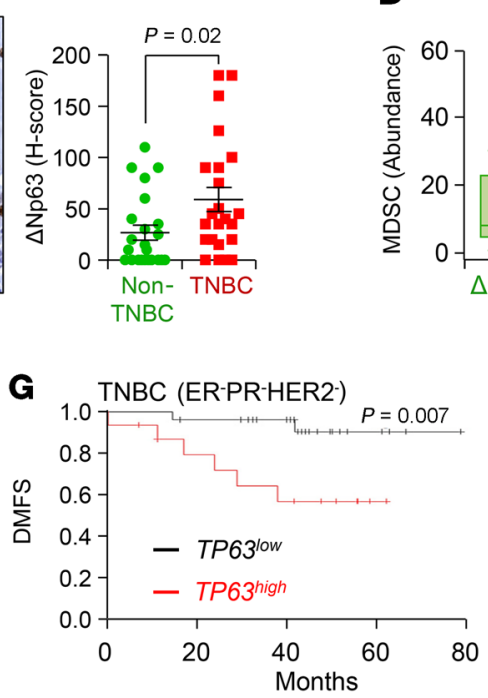

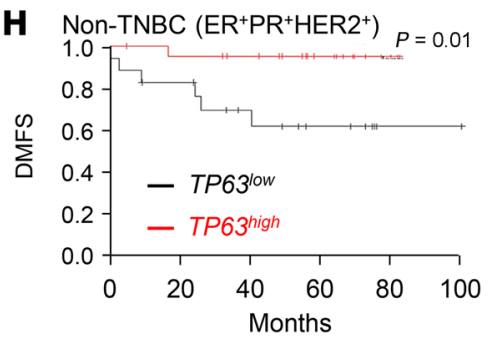

Figure 1. TNBC contains higher MDSC infiltration and high expression of $\triangle N$ p63, which is associated with reduced distant metastasis-free survival of human patients. (A and B) Representative immunofluorescence (IF) images (left) and calculated abundance (right) for CD33 and S100A9 (A) and CD15 and Lox-1 (B). Increased costaining of CD33 and S100A9 (yellow) in TNBC patients indicates increased MDSCs in these patients. Increased costaining of Lox-1 and CD15 (yellow cells indicated with white arrowheads) further confirms that MDSCs in TNBC are PMN-MDSCs. (C) Representative IHC images (left) and calculated $\mathrm{H}$-score (right) for $\triangle \mathrm{Np} 63$ expression in patient tissues. The $\mathrm{H}$-score value is the product of abundance of cells expressing respective protein (scale of $0-100$ ) multiplied by the intensity of expression of that protein (scale of $0-3$ ). ( $\mathbf{D}$ and $\mathbf{E}$ ) Box plot shows higher infiltration of MDSC (D) and PMN-MDSC (E) positivity in $\Delta$ Np63-high ( $\Delta \mathrm{N}^{\text {high }}$ ) than in $\Delta$ Np63-low ( $\Delta \mathrm{N}^{\text {low }}$ ) human TNBC tumor samples. $\Delta \mathrm{N}^{\text {high }}$ and $\Delta \mathrm{N}^{\text {low }}$ patients were stratified based on being above or below the median of $\Delta \mathrm{Np} 63 \mathrm{H}$-score in C. (A and B) Non-TNBC, $n=21$ patient samples; TNBC, $n=22$ patient samples. ( $\mathbf{D}$ and $\mathbf{E}$ ) TNBC, $n=22$ patient samples. (F-H) High p63 (TP63) expression correlates with reduced distant metastasis-free survival (DMFS) in ER-PR- (F), TNBC (C), but not non-TNBC (H) clinical samples in the KM Plotter breast cancer database (30). Scale bars: $40 \mu \mathrm{m}$ (A-C). (A-E) Mann-Whitney U test was used for scatter dot plots to quantify the difference between respective protein expressions. (F-H) Log-rank test was used for KM plots to calculate $P$ value.

growth from $\triangle$ Np63-KD HCC1806 (Figure 2, B and C), as well as reduced metastatic events of $\triangle \mathrm{Np} 63-\mathrm{KD}$ HCC1806 cells compared with the control (Figure 2, D and E, and Supplemental Figure 3B). This phenotype was also associated with reduced spleen size in mice injected with $\triangle$ Np63-KD HCC1806 cells (Supplemental Figure 3C). Similar findings were observed in an additional TNBC cell line, SUM159 (Supplemental Figure 3, D-G). Unlike HCC1806 cells, SUM159 cells form small microscopic metastatic lung nodules as seen by anti-GFP antibody staining (Supplemental Figure $3 \mathrm{H})$, which can be further augmented by resection of primary tumors. We clearly find that the macrometastasis-forming capability of SUM159 in lungs after resection is significantly reduced by $\Delta$ Np63-KD cells (Supplemental Figure 3I). To determine whether early stages of metastasis are affected by $\Delta \mathrm{Np} 63$ loss, we examined the disseminated tumor cells, also known as circulating tumor cells (CTCs), in blood of tumor-bearing mice. Short-term culture of СTCs from peripheral blood showed a significant decrease in the number of tumor cells in mice injected with $\triangle$ Np63-KD HCC1806 and SUM159 cells compared with their respective controls (Figure
2F and data not shown), a finding confirmed by the reduced ratio of human genomic DNA to mouse genomic DNA by quantitative PCR (Supplemental Figure 3, J-K). While we cannot rule out the possibility that the decreased numbers of CTCs are due to decreased tumor growth, these data are consistent with reduced CTC dissemination in $\triangle \mathrm{Np} 63-\mathrm{KD}$ TNBC cell-injected mice.

To further test the role of $\triangle \mathrm{Np} 63$ in metastasis, we injected control and $\triangle$ Np63-KD HCC1806 TNBC cells by tail vein and evaluated their ability to colonize and survive in distant organs (Figure 2G). HCC1806 TNBC metastasizes to several organs, including liver, lung, and lymph nodes (33), and we found that decreased levels of $\Delta \mathrm{Np} 63$ reduce metastases to all of these sites (Figure 2G and Supplemental Figure 4, A and B). In addition, we found that bone metastasis was dramatically reduced or completely absent in $\triangle$ Np63-KD HCC1806 cells (Supplemental Figure $4 \mathrm{C}$ ). In parallel, tail vein injection of control and $\triangle \mathrm{Np} 63-\mathrm{KD}$ SUM159 cells showed reduced lung metastasis in $\triangle \mathrm{Np} 63-\mathrm{KD}$ SUM159 TNBC cells (Supplemental Figure 4D), supporting a vital function of $\triangle \mathrm{Np} 63$ in metastasis. 
A

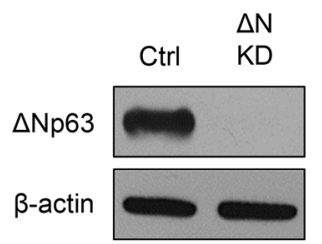

B
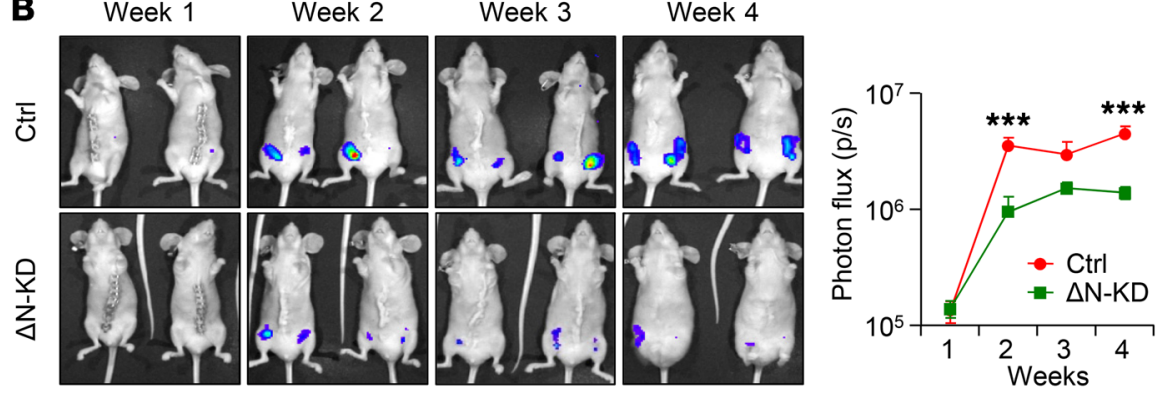

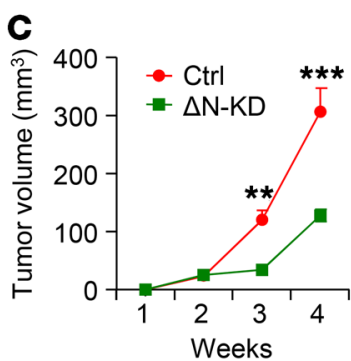

$\mathbf{F}$

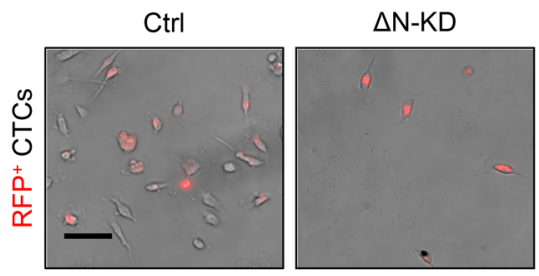

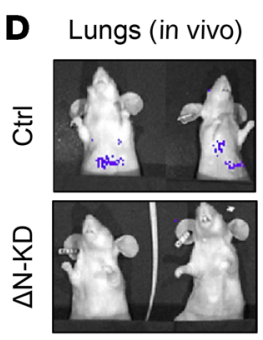
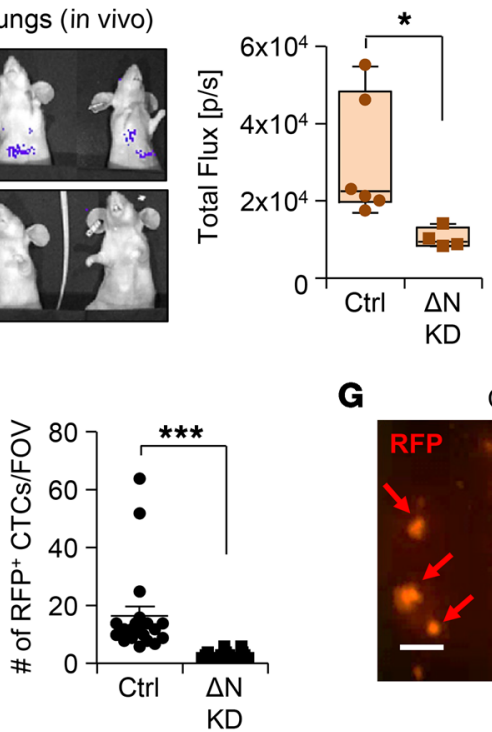
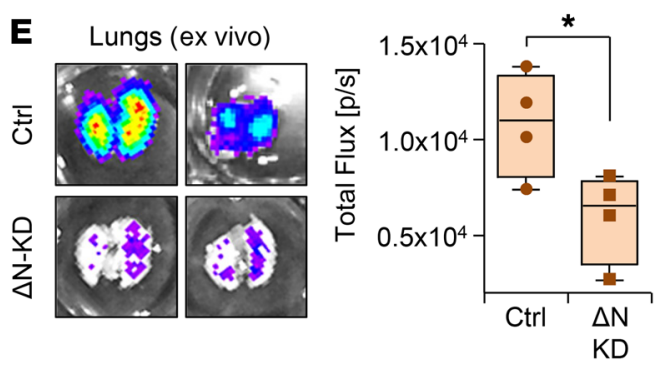

G Ctrl

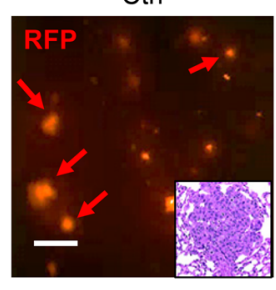

$\Delta \mathrm{N}-\mathrm{KD}$

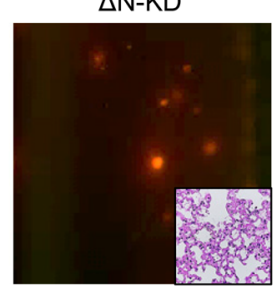

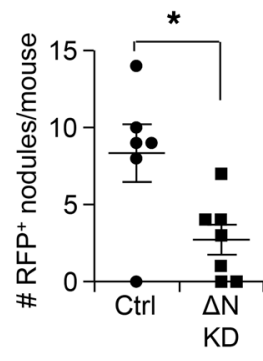

Figure 2. $\Delta$ Np63 promotes tumor growth, progression, and metastasis in human TNBC. (A) Western blot shows $\Delta N p 63(\Delta N)$ protein expression in control and $\triangle$ Np63-KD HCC1806 cells after shRNA lentivirus vector-mediated knockdown (KD). (B) Red fluorescence (RFP) and Renilla luciferase-expressing control and $\triangle \mathrm{Np} 63-\mathrm{KD}$ HCC1806 cells $\left(2 \times 10^{5}\right)$ were injected into the mammary fat pad of nude mice. Representative mouse images (left) and tumor growth (right; total flux, photons per second $[\mathrm{p} / \mathrm{s}]$ ) show bioluminescent signal from tumors in vivo. (C) Tumor growth curves show data of palpated tumor growth of indicated groups. (B and $\mathbf{C}$ ) $n=10$ tumors per group. Contralateral mammary glands (4th position) of $n=5$ mice per group were used for injection. (D and E) Control and $\triangle$ Np63-KD HCC1806 tumor-bearing mice show in vivo (D) and ex vivo (E) lung metastasis by bioluminescence imaging. Ctrl, $n=6$ mice per group; $\Delta \mathrm{N}-\mathrm{KD}, n=4$ mice per group. (F) Bright-field and fluorescence images show RFP+ circulating tumor cells (CTCs) after culture. CTCs were isolated from blood of control and $\triangle \mathrm{Np63-KD} \mathrm{HCC1806} \mathrm{xenograft-bearing} \mathrm{mice} \mathrm{and} \mathrm{cultured} \mathrm{for} 1$ week. $n=5$ mice per group. (C) HCC1806 cells ( $\left.2 \times 10^{5}\right)$ were injected into the bloodstream of NSG mice via tail vein to study metastasis; a representative image of RFP+ lung metastatic nodules is shown (G, left, red arrows). Quantification of metastatic RFP+ nodules of indicated groups is shown at right. The insets show H\&E images of sections of lung, showing metastatic nodule in control. Ctrl, $n=6$ mice; $\Delta \mathrm{N}-\mathrm{KD}, n=7$ mice. (B and C) Two-way ANOVA was performed with Bonferroni post-test adjustment. (D-G) Mann-Whitney $U$ test was used to compute $P$ values. Scale bars: $40 \mu \mathrm{m}(\mathbf{F})$ and $500 \mu \mathrm{m}(\mathbf{C})$. Data are presented as the mean \pm SEM from 3 independent experiments. ${ }^{*} P<0.05,{ }^{* *} P<0.01,{ }^{* *} P<0.001$. FOV, field of view.

Loss of $\triangle N p 63$ leads to dramatic alteration of tumor cell fate and reduced proliferation, immune infiltrate, and angiogenesis in TNBC. Breast cancer growth and progression are regulated by a complex interplay between tumor cells and the TME, the latter of which has a dramatic impact on tumor growth, progression, and metastasis (5-7). This interaction is reciprocal, as cancer cells also shape the TME for tumor and metastasis-promoting effects. Notably, H\&E staining analysis of the primary tumors generated by HCC1806 and SUM159 TNBC cells revealed massive infiltration of leukocytes to the control xenograft tumors in comparison with the tumors from $\triangle$ Np63-KD HCC1806 cells and $\triangle$ Np63-KD SUM159 cells (Supplemental Figure 5, A and B), which were further confirmed with staining with CD 45 antibody (Supplemental Figure 5, C and D). We also observed reduced $\mathrm{K}^{+} 4^{+}$cells in $\triangle \mathrm{Np} 63-\mathrm{KD}$ TNBCs compared with control, suggesting that $\Delta \mathrm{Np} 63$ is an important basal cell fate regulator (Supplemental Figure 5, E and F). Furthermore, reduced tumor cell proliferation (marked by Ki67) was observed in tumors from $\triangle$ Np63-KD HCC1806 cells and $\Delta$ Np63-KD SUM159 cells compared with control cells, suggesting that reduced proliferation may be responsible for reduced tumor growth in $\triangle \mathrm{Np} 63-\mathrm{KD}$ cells (Supplemental Figure 5, G and H).

Angiogenesis positively correlates with metastasis, and $\Delta \mathrm{Np} 63$ promotes tumor angiogenesis in pediatric neuroblastoma, osteosarcoma, and squamous cell carcinoma (34-36). Therefore, we assessed the expression of CD31 and CD34, markers for angiogenesis, as well as smooth muscle actin (SMA) (37) in HCC1806 and SUM159 primary tumors. The number of $\mathrm{CD} 34^{+}$and $\mathrm{CD} 31^{+}$blood vessels (as a measure of microvascular density [MVD]) was signifi- 
A

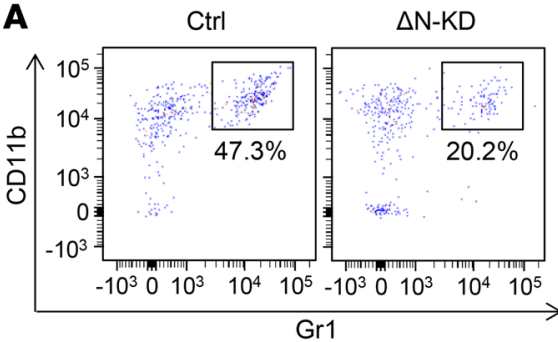

G

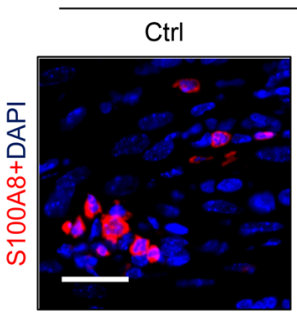

HCC1806 (Primary tumor)
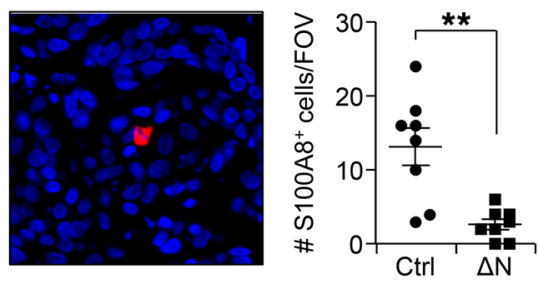

$\mathrm{KD}$

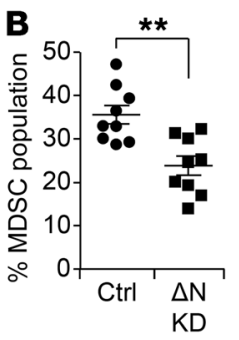

$\mathrm{KD}$

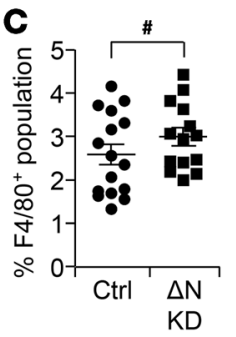

H

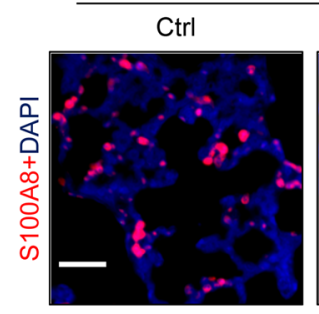

HCC1806 (Metastatic site)
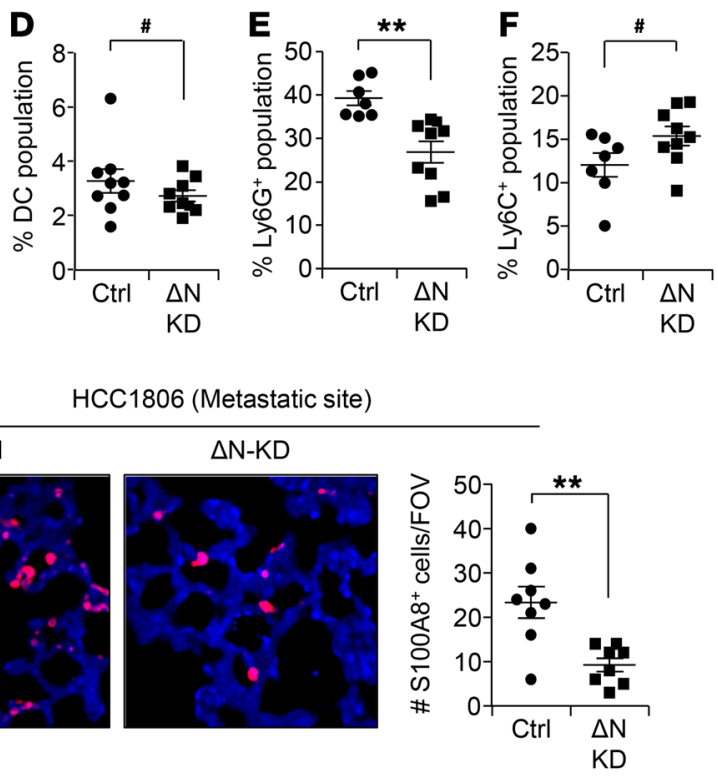

I

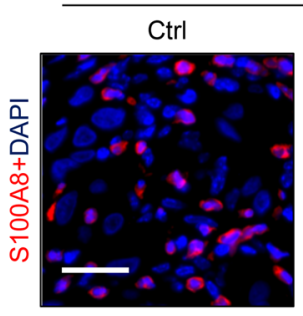

SUM159 (Primary tumor)

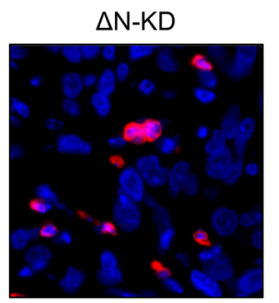

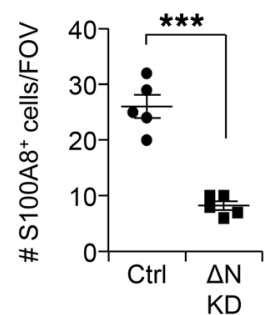

J SUM159 Lung (Metastatic site)

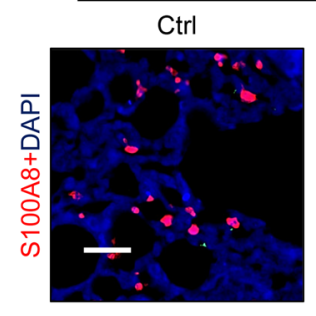
$\Delta \mathrm{N}-\mathrm{KD}$
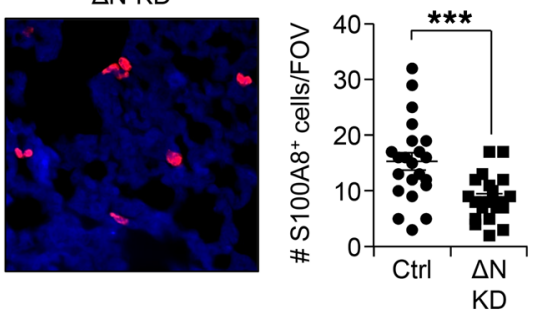

Figure 3. $\triangle \mathrm{Np63}$ promotes recruitment of MDSCs at primary tumor and metastatic site. (A) Flow profile of CD45 population of control and $\triangle N p 63-K D$ HCC1806 tumor based on the staining of CD11B and Gr1 markers shows MDSC population. Fluorescence Minus One (FMO) negative control is shown in Supplemental Figure 7A. (B-D) Flow cytometric analysis depicts percentage of MDSCs (CD11B+C $\left.r^{1+} \mathrm{F} 4 / 80^{-}\right)(\mathbf{B})$, macrophages (CD45+F4/80+) (C), and dendritic cells (DCs; CD45 + CD11c $\left.^{+}\right)(\mathbf{D})$ in primary tumors of indicated groups. (E and F) Flow cytometric analysis depicting MDSC subpopulations: PMN-MDSCs

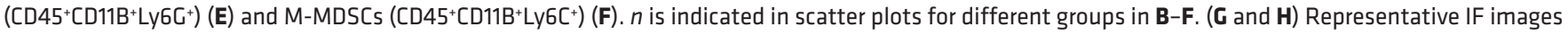
show S100A8 ${ }^{+}$stained myeloid cells at the primary tumor (C) and metastatic site $(\mathbf{H})$ of control and $\triangle$ Np63-KD HCC1806 cells. Scatter plots to the right show quantification of S100A8 ${ }^{+}$cells per FOV. (I and J) IF images show myeloid cells in primary tumor (I) and at metastatic site (J) of control and $\triangle N$ N63-KD SUM159 cells. Scatter plots to the right show quantification of S100A8 ${ }^{+}$cells. Scale bars: $40 \mu \mathrm{m}(\mathbf{G}-\mathbf{J}) .{ }^{* *} P<0.01,{ }^{* * *} P<0.001$, ${ }^{*} \mathrm{NS}$. Data are presented as the mean \pm SEM. (B-J) Student's $t$ test was used to compute $P$ values. (G-J) $n=3$ samples were used, and several random fields were evaluated per sample for quantification from 3 independent experiments.

cantly reduced in $\triangle \mathrm{Np} 63-\mathrm{KD}$ tumors compared with control TNBC tumors (Supplemental Figure 6, A-D). Evaluation of microvascular integrity (MVI) using $\mathrm{CD} 34^{+} / \mathrm{SMA}^{+}$or $\mathrm{CD} 31^{+} / \mathrm{SMA}^{+}$costaining of blood vessels revealed reduced $\mathrm{CD} 34^{+} \mathrm{SMA}^{+}$or $\mathrm{CD}^{+} 1^{+} \mathrm{SMA}^{+}$(double-positive) blood vessels in TNBC tumor xenografts (Supplemental Figure 6, A-H), indicating poor vessel quality that could potentially lead to intra/extravasation of tumor cells that could permit metastasis. Notably, loss of $\triangle \mathrm{Np} 63$ led to an increase in CD34+S$\mathrm{MA}^{+}$or CD31 ${ }^{+} \mathrm{SMA}^{+}$(double-positive; white arrowheads) blood vessels compared with control (Supplemental Figure 6, A-H), indicating that $\triangle \mathrm{Np} 63$ may regulate both MVD and MVI in TNBC. Taken together, our studies demonstrate that $\Delta$ Np63 influences leukocyte recruitment, proliferation of tumor cells, cell fate, and angiogenesis, all of which could contribute to the aggressive nature of TNBC.

$\triangle N p 63$ promotes recruitment of MDSCs at the primary tumor and metastatic sites. Given the strong positive correlation between $\triangle$ Np63 expression and PMN-MDSC infiltration in TNBC patient samples (Figure 1, A-E), we next analyzed the immune infiltrates in control and $\triangle$ Np63-KD xenograft tumors of TNBC. Flow cytometric analysis using CD45, CD11B, and Gr1 antibodies revealed that the $\mathrm{CD} 45^{+}$immune cell infiltrate was predominantly MDSCs (Figure 3, A and B) and that it was significantly reduced in $\triangle \mathrm{Np} 63-\mathrm{KD}$ tumors compared with control. In contrast, no significant difference was observed between macrophages $\left(\mathrm{CD} 45^{+} \mathrm{F} 4 / 80^{+}\right)$and dendritic cells $\left(\mathrm{CD}^{2} 5^{+} \mathrm{CD} 11 \mathrm{c}^{+}\right)$(Figure 3, C and D). Further characterization of tumor-infiltrating MDSCs using Ly6G and Ly6C antibodies indicated that the affected MDSCs were Ly6G ${ }^{+}$PMN-MDSCs

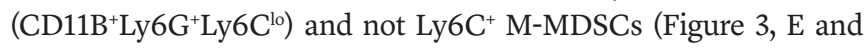
F), supporting human patient IHC data (Figure 1, A and B). Additionally, we found that $\triangle$ Np63 promotes PMN-MDSC infiltration at metastatic sites such as the lung (Supplemental Figure 7, A-G). Similarly to the primary tumor, other immune populations remain unchanged at lung metastatic sites in tumor-bearing mice from $\triangle$ Np63-KD HCC1806 injected cells compared with control (Supplemental Figure 7, D and E). Peripheral blood analysis indicated reduced numbers of MDSCs in $\triangle \mathrm{Np} 63-\mathrm{KD}$ tumor-injected mice 
A

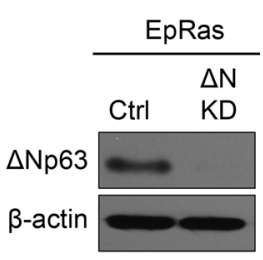

B

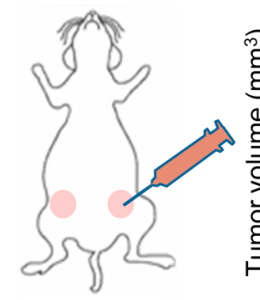

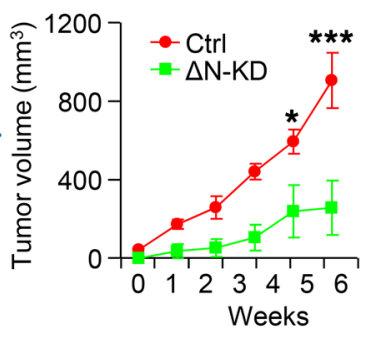

C

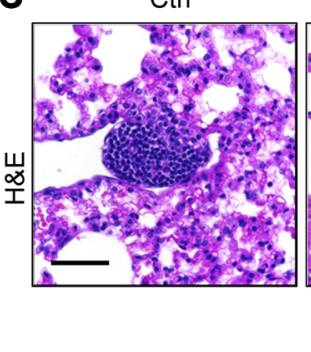

$\triangle \mathrm{N}-\mathrm{KD}$

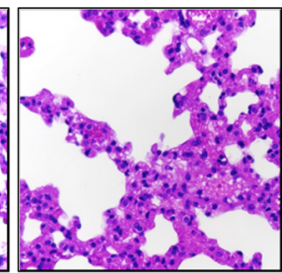

D

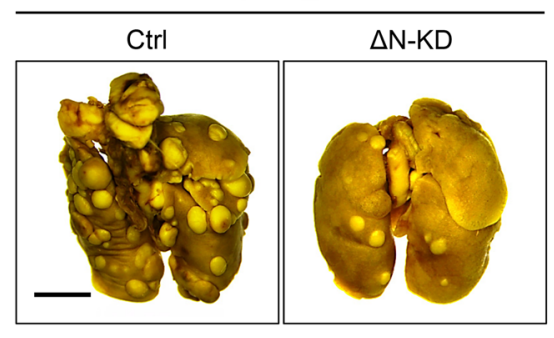

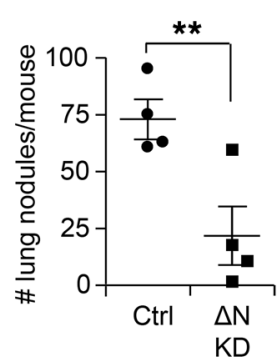

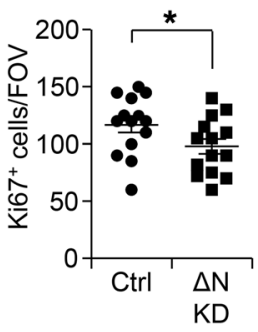

$\mathbf{F}$

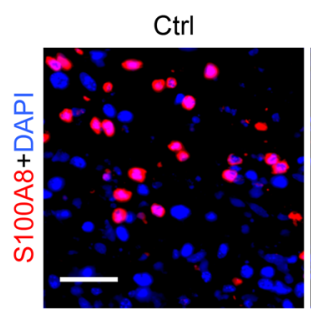

Primary tumor

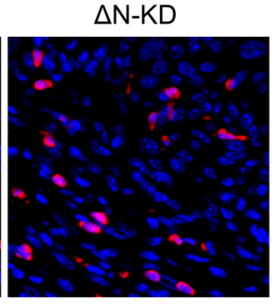

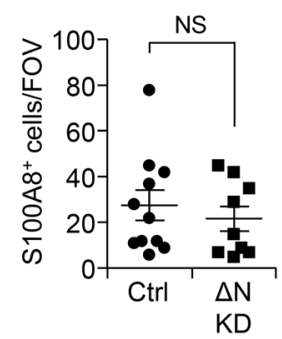

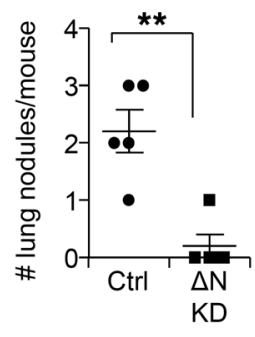

E

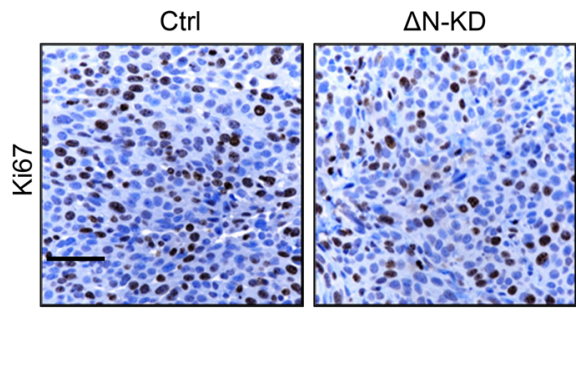

G

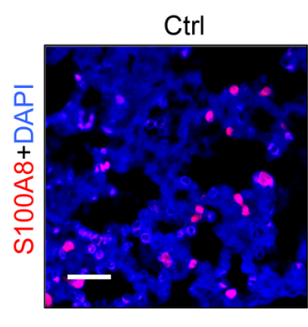

Lung (Metastatic site)

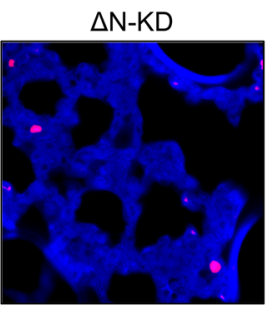

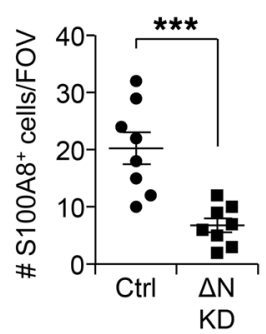

Figure 4. $\Delta$ Np63 promotes tumor growth, progression, and metastasis in mouse TNBC. (A) Western blot shows $\Delta$ Np63 ( $\Delta N$ ) protein expression in EpRas cells after shRNA lentivirus vector-mediated knockdown (KD). (B) EpRas control and $\Delta$ Np63-KD EpRas cells (5 $\left.\times 10^{4}\right)$ were injected into the mammary fat pad of BALB/c mice. Schematic shows experimental approach (left) and tumor growth curve (right). Ctrl, $n=8$ tumors; $\Delta \mathrm{N}-\mathrm{KD}, n=6$ tumors. (C) Representative $H \& E$ images of lung show metastatic nodule of indicated groups. $n=5$ mice per group. (D) EpRas cells $\left(2 \times 10^{4}\right)$ were injected into the bloodstream of BALB/c mice via tail vein (TV) to study metastasis. Representative images show lung nodules (left), and scatter plot (right) represents quantification. (E) Representative IHC images show the expression of cell proliferation (Ki67) in control and $\Delta$ Np63-KD EpRas primary tumors. Scatter plots (right) represents quantification. (F and $\mathbf{G})$ Representative IF images show myeloid cell infiltration (S100A8) in the primary tumor (F) and lung metastatic site (G). Scatter plots (right) represent quantification of S100A8+ myeloid cells. (B) Two-way ANOVA was performed followed by Bonferroni post-test to compute $P$ value. (C) Mann-Whitney $U$ test was used to compute $P$ value. (D-G) Student's $t$ test was used to compute $P$ values. Scale bars: $40 \mu m(\mathbf{C}$ and $\mathbf{E}-\mathbf{G})$ and $500 \mu \mathrm{m}$ (D). Data are presented as the mean \pm SEM. ${ }^{*} P<0.05,{ }^{* *} P<0.01,{ }^{* *} P<0.001$, ${ }^{*} \mathrm{NS}$. (E-G) $n=3$ samples were used, and several random fields per sample were evaluated for quantification from 3 independent experiments.

compared with control (Supplemental Figure 7H), suggesting that loss of $\triangle \mathrm{Np} 63$ reduces recruitment of MDSCs from bone marrow. These results were further confirmed by staining of primary tumor and metastatic lung with the myeloid marker S100A8 antibody, which demonstrated that myeloid cells were reduced in $\triangle \mathrm{Np} 63-\mathrm{KD}$ primary and metastatic tumors from HCC1806 (Figure 3, G and H) and SUM159 cells (Figure 3, I and J) compared with control.

As PMN-MDSCs phenotypically resemble neutrophils (24), we next confirmed that the $\mathrm{CD} 45^{+} \mathrm{CD} 11 \mathrm{~B}^{+} \mathrm{Gr} 1^{+}$populations we identified represent bona fide MDSCs by performing in vitro T cell proliferation assays - an established functional test for MDSCs. Our data clearly show reduced $\mathrm{T}$ cell proliferation following addition of $\mathrm{CD}_{4} 5^{+} \mathrm{CD} 11 \mathrm{~B}^{+} \mathrm{Gr} 1^{+}$MDSCs from the primary mammary tumor and spleen (Supplemental Figure 7I) of tumor-bearing mice. Moreover, MDSCs isolated from these xenograft tumors suppressed $\mathrm{T}$ cell function, as seen by reduced intracellular IFN- $\gamma$ expression in $\mathrm{T}$ cell coculture assays (Supplemental Figure 7, J and K). Finally, H\&E analysis showed that these MDSCs exhibit an immature granulocytic morphology similar to that of PMN-MDSCs (Supplemental Figure 7L). Taken together, our data indicate that PMN-MDSCs are specifically recruited to primary and metastatic lung TNBC tumors in a $\triangle \mathrm{Np} 63$-dependent manner.

Loss of $\triangle N p 63$ reduces primary tumor growth and metastasis in mouse TNBC cells. To rigorously test the function of $\triangle N p 63$ in tumor growth and metastasis, we next extended our study to a mouse breast cancer cell line that could be evaluated in syngeneic 
A Breast Basal vs. Luminal Up B

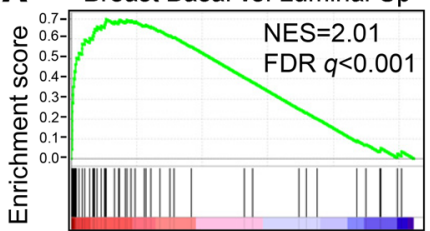

Ctrl vs. $\triangle \mathrm{Np} 63-\mathrm{KD}$

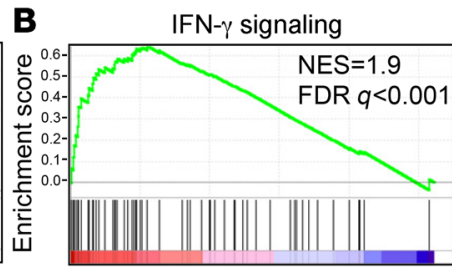

Ctrl vs. $\triangle$ Np63-KD
C

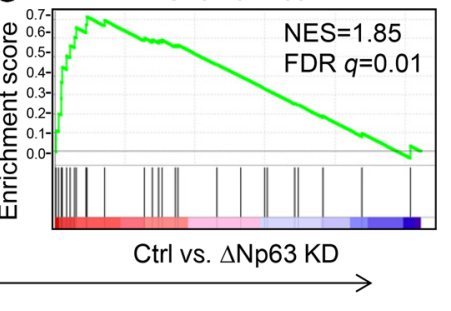

D $\quad$ HCC1806 SUM159

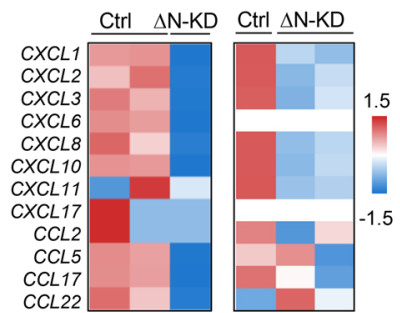

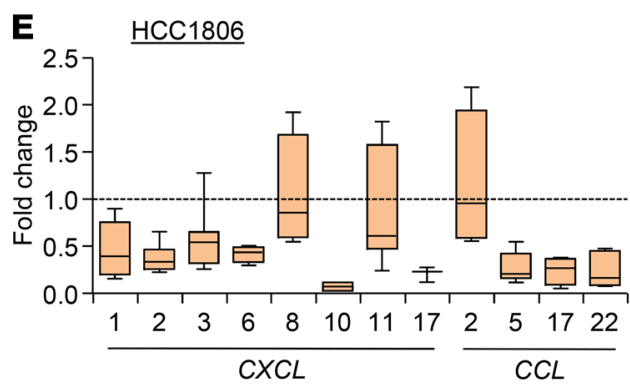

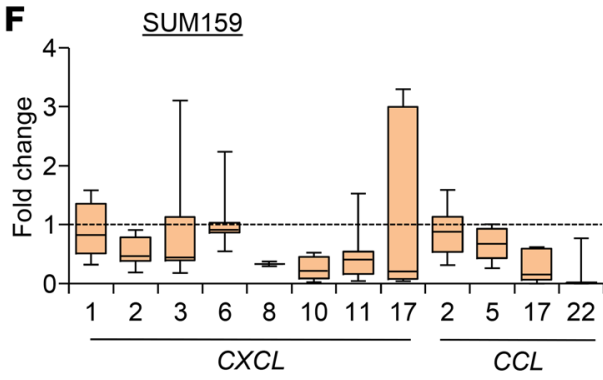

G
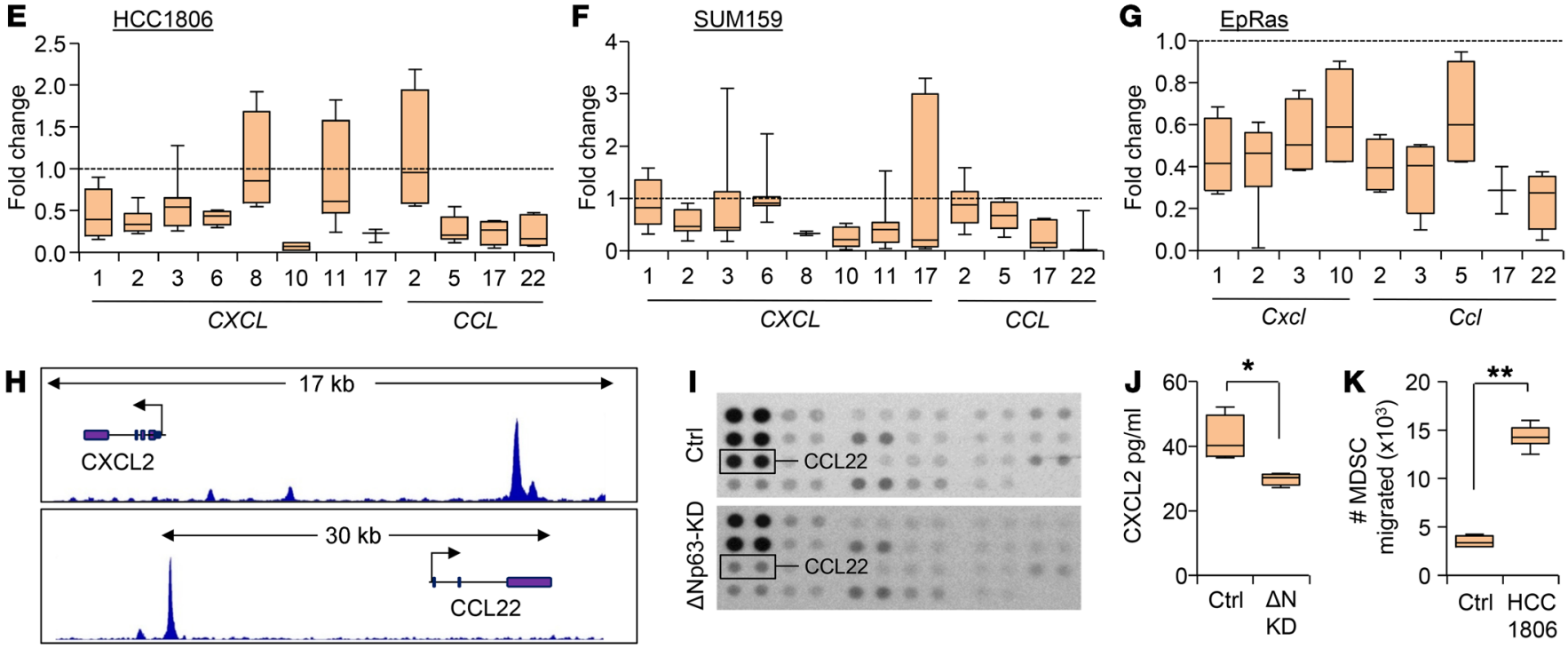

I

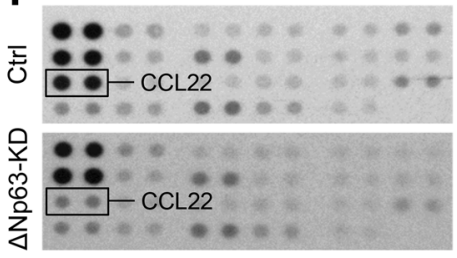

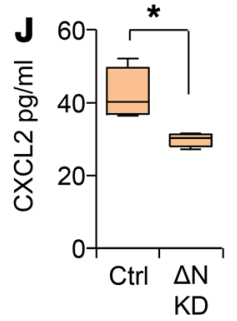

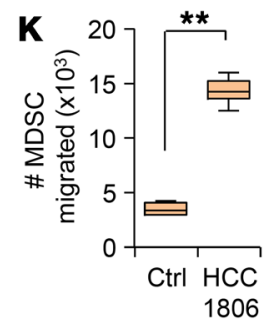

L

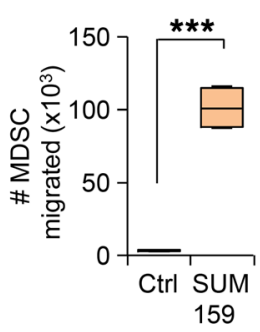

M

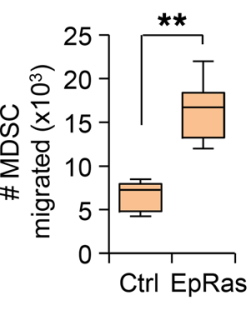

$\mathbf{N}$

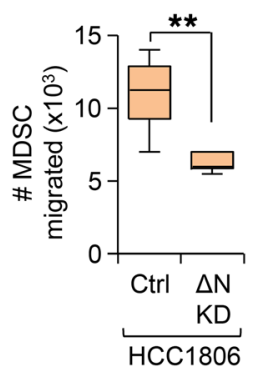

0

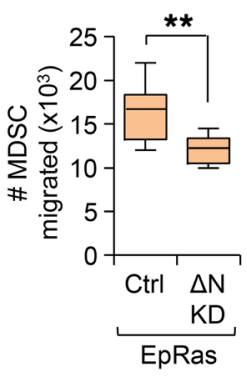

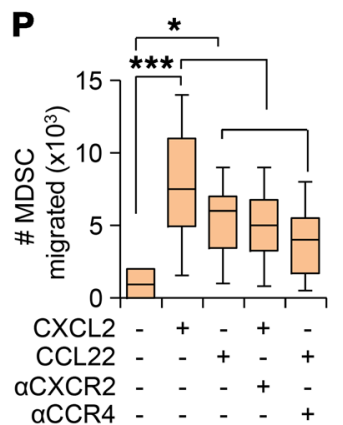

Q

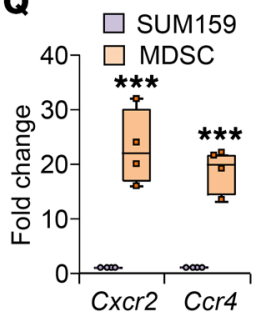

Figure 5. $\Delta$ Np63 directly regulates CXCL2 and CCL22, promoting MDSC recruitment in TNBC tumors. (A-C) Cene set enrichment analysis demonstrating enriched basal signature compared with luminal signatures (A), enriched IFN signaling gene signature (B), and enriched chemokine signature (C) in control compared with $\triangle$ Np63-KD HCC1806 cells. NES, normalized enrichment score. (D) Heatmaps depicting relative expression of $\Delta$ Np63-dependent chemokines in control and $\triangle \mathrm{Np63-KD}$ of HCC1806 and SUM159 cells. (E-G) Quantitative reverse transcriptase PCR (RT-qPCR) analysis of indicated chemokines in control and $\triangle$ Np63-KD HCC1806 (E), SUM159 (F), and EpRas cells (G). The relative expression of control was considered fold change 1, depicted by the dotted lines. (H) ChIP-Seq analysis shows the putative $\triangle$ Np63-binding site (peaks) in the human CXCL2 and CCL22 gene loci using $\triangle N p 63$ antibody. (I) Representative images of chemokine antibody arrays from control and $\triangle$ Np63-KD HCC1806 cells. (J) The box plot shows the quantification of protein level of CXCL2 by ELISA in conditioned media of control and $\triangle$ Np63-KD HCC1806 cells. (K-M) Quantification of migrated MDSCs toward conditioned media of indicated cell lines compared with control (plain medium was added). ( $\mathbf{N}$ and $\mathbf{0}$ ) Quantification of migrated MDSCs toward conditioned media of control and $\triangle$ Np63KD cells (HCC1806 and EpRas). (P) Quantification of migrated MDSCs toward indicated chemokine ligand or chemokine receptor inhibitor. (Q) RT-qPCR analysis shows the quantification of mRNA expression levels of indicated chemokine receptors in MDSCs compared with TNBC cells. SUM159 tumor cells were used for analysis. (A-Q) $n=3$ independent experiments performed in technical duplicate. Real-time PCR values were normalized to the housekeeping gene GAPDH (mouse and human), and data are presented as the mean \pm SD. (E-G, J-O, and $\mathbf{Q}) P$ values were calculated using Student's $t$ test. ( $\mathbf{P}) P$ value was calculated using 1-way ANOVA with Tukey's multiple-comparisons post hoc test. ${ }^{*} P<0.05,{ }^{* *} P<0.01,{ }^{* * *} P<0.001$.

immune-competent BALB/c mice. The EpRas cell line is derived from mouse $\mathrm{EpH} 4$ cells, which constitutively express oncogenic Ras and are $\mathrm{ER}^{-/ 10}$ and $\mathrm{PR}^{-/ 10}$ (ref. 38 and Supplemental Figure 8A). We generated 2 independent shRNAs that specifically target mouse $\triangle \mathrm{Np63}$ and behaved similarly in functional assays (21) and contin- ued with 1 knockdown construct (referred to as $\Delta \mathrm{N}-\mathrm{KD}$ ) (Figure $4 \mathrm{~A}$ and Supplemental Figure $8 \mathrm{~B}$ ). Knockdown of $\Delta \mathrm{Np} 63$ had no effect on TAp63 in the EpRas cells (Supplemental Figure 8B). Similar to previous results with human TNBC cells (Figure 2), we found significant reduction in primary tumor growth (Figure 4B), mouse spleen 
size (Supplemental Figure 8C), and spontaneous lung metastasis (Figure 4C) in $\triangle$ Np63-KD EpRas cells compared with control. We found a significant decrease in the number of CTCs in the peripheral blood of mice injected with $\triangle$ Np63-KD EpRas cells compared with control (Supplemental Figure 8D), supporting earlier data with human breast cancer cells (Figure 2F). Moreover, tail vein injection of $\triangle \mathrm{Np} 63-\mathrm{KD}$ EpRas cells generated significantly fewer lung metastatic nodules compared with control cells (Figure 4D). Extensive analysis of the primary tumor demonstrated decreased expression of the basal marker (K14) and increased expression of the luminal marker, K8, in $\triangle \mathrm{Np63-KD} \mathrm{EpRas} \mathrm{cells} \mathrm{(Supplemental}$ Figure 8, E-H, and data not shown), similar to our observations in human TNBC xenograft models. $\triangle$ Np63-KD tumors also showed a significant decrease in Ki67 expression compared with control tumors (Figure 4E). As there was no significant difference in proapoptotic cleaved caspase- 3 expression between control and $\Delta$ Np63-KD tumors (data not shown), decreased proliferation likely accounts for the slower tumor growth. Angiogenesis (both MVD and MVI) was altered in $\triangle \mathrm{Np} 63-\mathrm{KD}$ tumors compared with control tumors (Supplemental Figure 8, I-L), indicating an indispensable role for $\triangle \mathrm{Np} 63$ in TNBC tumor angiogenesis. Finally, quantitative PCR on $\triangle \mathrm{Np63-KD}$ tumors demonstrated reduced levels of angiopoietin 2, an important factor for neovascularization (39) (Supplemental Figure 8M), in comparison with control tumors.

Histological analyses demonstrated that $\triangle \mathrm{Np} 63-\mathrm{KD}$ tumors had fewer CD45 leukocytes than controls (Supplemental Figure 9A), corroborating earlier human xenograft tumors. Finally, immunofluorescence analysis confirmed that these immune cells were $\mathrm{S} 100 \mathrm{~A}^{+}$myeloid cells and that they were reduced in $\triangle$ Np63-KD primary and metastatic tumors compared with control (Figure 4, $\mathrm{F}$ and $\mathrm{G}$ ). Reduction of $\mathrm{S} 100 \mathrm{~A} 8^{+}$cells in primary tumors was further confirmed by quantitative PCR (Supplemental Figure 9B). Additionally, we found that $\triangle \mathrm{Np} 63$ promotes PMN-MDSC $\left(\mathrm{CD} 11 \mathrm{~B}^{+} \mathrm{Ly}_{6 \mathrm{G}}{ }^{+} \mathrm{Ly}_{6} \mathrm{C}^{\mathrm{lo}}\right)$ infiltration at primary tumor and metastatic lung (Supplemental Figure 9, C-N), while other immune populations, including $\mathrm{T}$ cells and $\mathrm{NK}$ cells, remain unchanged (Supplemental Figure 9, C, E-I, and K-N). These data indicate that loss of $\triangle \mathrm{Np} 63$ alters the TME of TNBCs by reducing the number of MDSCs (independent of T cell and NK cell suppression) and angiogenesis, which may lead to reduced metastasis.

We next evaluated whether the protumorigenic function of $\triangle \mathrm{Np63}$ is restricted to TNBCs. For this, we overexpressed $\Delta \mathrm{Np} 63$ in WTB cells derived from an MMTV-PyMT-derived luminal breast cancer model $(40,41)$, which have low endogenous levels of $\Delta$ Np63 (Supplemental Figure 10, A and B). Notably, this overexpression did not alter tumor growth significantly (Supplemental Figure 10C). However, expression of the basal cell marker K14 increased in $\triangle$ Np63-overexpressing WTB tumor cells compared with control (Supplemental Figure 10, D and E), supporting its function as a strong driver of basal cell fate. Taken together, these data suggest that $\triangle \mathrm{Np} 63$ specifically promotes TNBC tumor growth, progression, and metastasis in both immune-competent and immunocompromised mice and that its loss reduces MDSC infiltration and angiogenesis.

$\triangle N p 63$ directly regulates CXCL2 and CCL22 to promote MDSC recruitment in TNBC. To investigate the molecular mechanism for $\triangle$ Np63-dependent recruitment of MDSCs, we performed RNASeq experiments with HCC1806 and SUM159 control and $\Delta$ Np63-
KD cell lines. Corroborating our IHC data (Supplemental Figure 5, $\mathrm{E}$ and $\mathrm{F}$ ), gene set enrichment analyses showed a decreased basal cell signature in $\triangle \mathrm{Np} 63-\mathrm{KD}$ TNBC cells compared with control (Figure 5A). Additionally, we identified several genes belonging to the IFN and chemokine signaling pathways that were significantly downregulated in $\Delta$ Np63-KD HCC1806 and $\Delta$ Np63-KD SUM159 cells compared with controls (Figure 5, B and C). Chemokines promote MDSC infiltration in multiple cancers, including breast cancer $(42,43)$, and regulate cancer cell proliferation and metastasis, and their expression is associated with increased angiogenesis, a strong indicator of tumor invasiveness and metastasis (42, 43). Hence, we focused on chemokines (CXCL2, CXCL3, CXCL1O, CCL5, CCL17, and CCL22) that were differentially expressed in $\triangle$ Np63-KD HCC1806 and $\triangle$ Np63-KD SUM159 compared with control cells (Figure 5D) and/or differentially expressed in human and mouse TNBC cell lines (Figure $5, E-G$ ) with reduced $\Delta$ Np63 compared with control. Examination of $\Delta \mathrm{Np} 63 \mathrm{ChIP}$-Seq data from HCC1806 cells revealed several p63-binding sites in the enhancer regions of 2 of these chemokines, CXCL2 and CCL22 (Figure $5 \mathrm{H})$, strongly indicating that they are direct targets of $\Delta \mathrm{Np} 63$. As chemokines are often secreted from the tumor to recruit MDSCs, we next determined whether these $\Delta$ Np63-dependent chemokines were secreted using chemokine array blots and ELISA (Figure 5, I and J). Both CXCL2 and CCL22 were significantly decreased in media supernatants from $\triangle \mathrm{Np} 63-\mathrm{KD}$ cells compared with the control (Figure 5, I and J, and data not shown), confirming loss of functional protein for these chemokines in $\triangle$ Np63-KD TNBCs. To evaluate the relationship between $\triangle \mathrm{Np} 63$ and CXCL2/CCL22 in the clinical setting, we interrogated a human breast cancer TCGA data set (30) and found that CXCL2 and CCL22 expression positively correlate with TP63 in ER-PR-HER2- TNBC patients but not in non-TNBC patients (Supplemental Figure 11, A and B, and data not shown). Consistently, we found that high levels of CXCL2 correlate with reduced DMFS in TNBC patients $(P=0.05)$, but not in non-TNBC samples (Supplemental Figure 11, $C$ and E). TNBC patients expressing CCL22 showed a similar trend $(P=0.406$; Supplemental Figure 11D); however, the data did not reach statistical significance because of a relatively small sample size of metastatic TNBC patients in this data set. Interestingly, higher CCL22 levels are significantly associated with better patient survival in non-TNBC patients ( $P=0.002$; Supplemental Figure $11 \mathrm{~F})$, suggesting a subtype-specific effect of CCL22. Taken together, our data demonstrate that CXCL2 and CCL22 are direct targets of $\triangle \mathrm{Np} 63$ in TNBCs and may contribute to poor TNBC patient survival.

To verify that migration/recruitment of MDSCs is promoted by $\Delta$ Np63-dependent chemokines, we performed in vitro chemotaxis assays using supernatants from HCC1806, SUM159, and EpRas (human and mouse TNBC) cells. A strong migratory activity was observed in mouse MDSCs $\left(\mathrm{CD} 11 \mathrm{~B}^{+} \mathrm{Gr} 1^{+}\right)$in response to supernatants from both human (HCC1806 and SUM159) and mouse (EpRas) cell lines compared with media control (Figure 5, $\mathrm{K}-\mathrm{M})$. Notably, this effect was dependent on $\Delta \mathrm{Np} 63$, as $\Delta \mathrm{Np} 63-\mathrm{KD}$ HCC1806 (human) and $\Delta$ Np63-KD EpRas (mouse) cells failed to promote migratory activity compared with control cells (Figure 5, $\mathrm{N}$ and $\mathrm{O}$ ). Additional MDSC chemotaxis assays using mouse splenic or tumor MDSCs and purified human CXCL2 and CCL22 ligands revealed a significant increase in MDSC migration with 
A

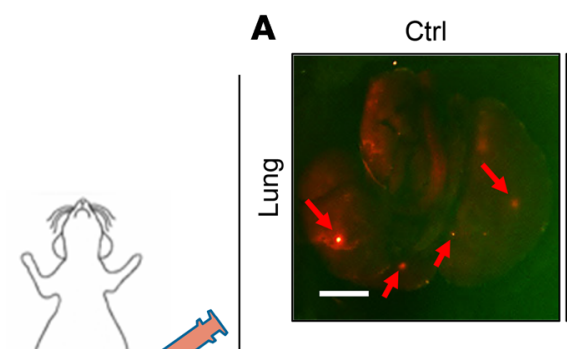

B

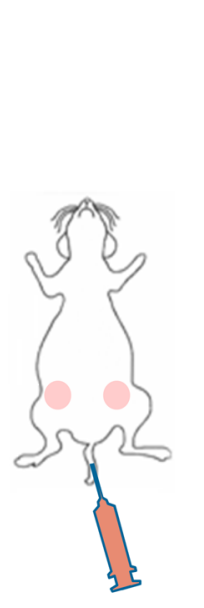

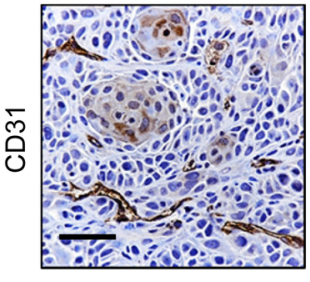

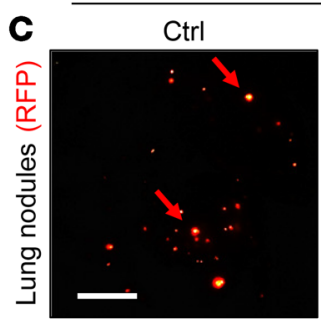

$\Delta \mathrm{N}-\mathrm{KD}$

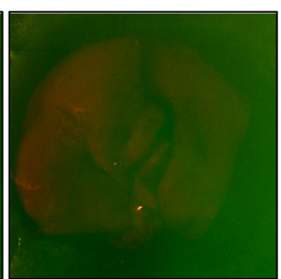

$\triangle \mathrm{N}-\mathrm{KD}$

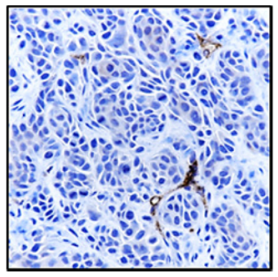

aCXCR2 SB 225002

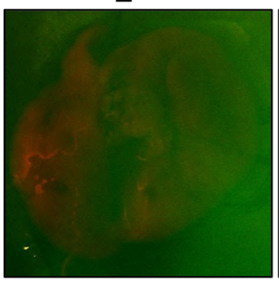

aCXCR2_SB 225002
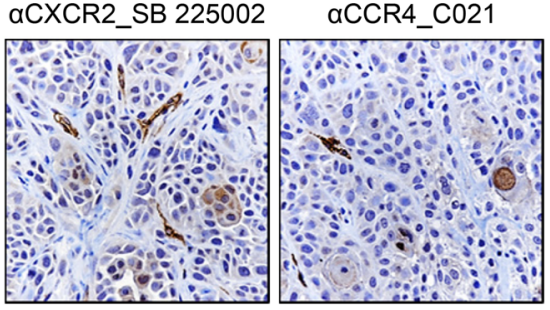

Lung (Metastatic site)
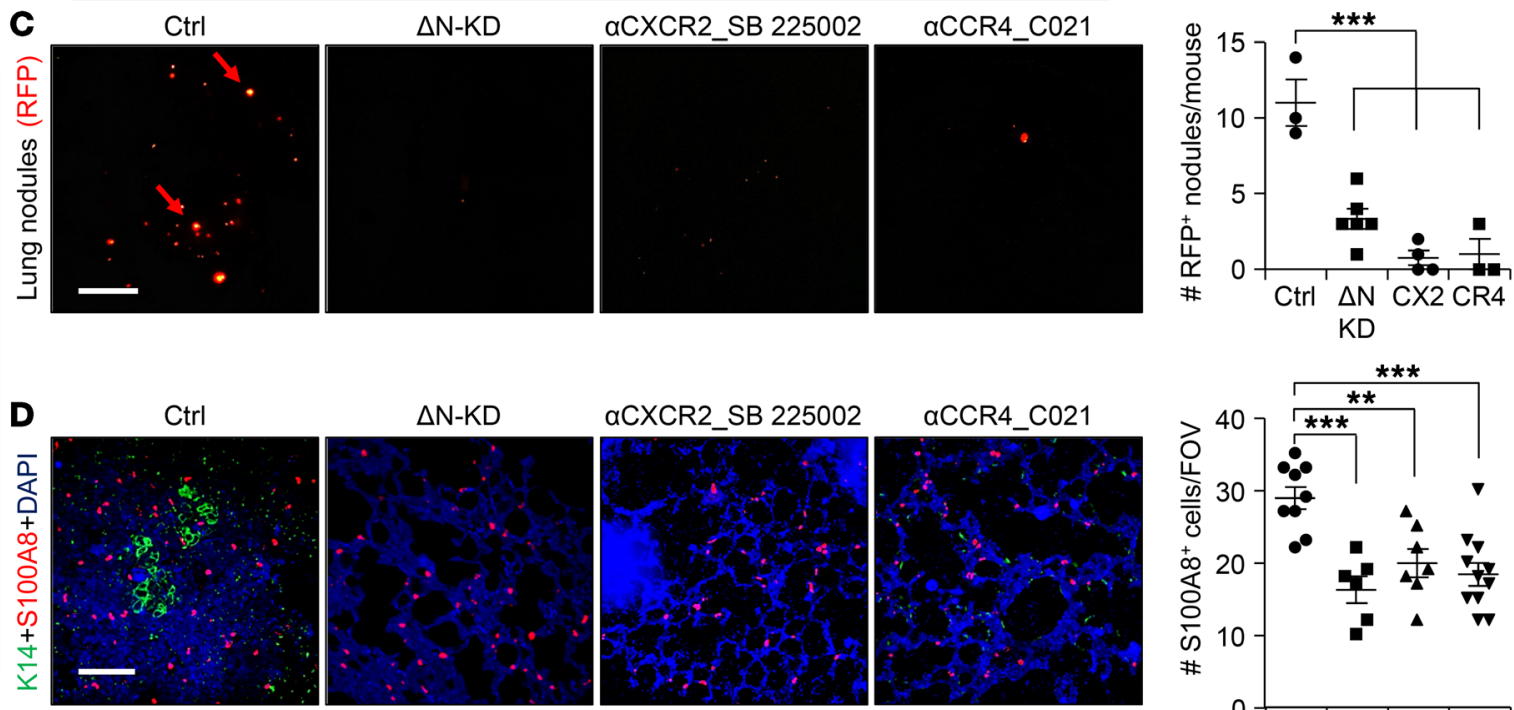
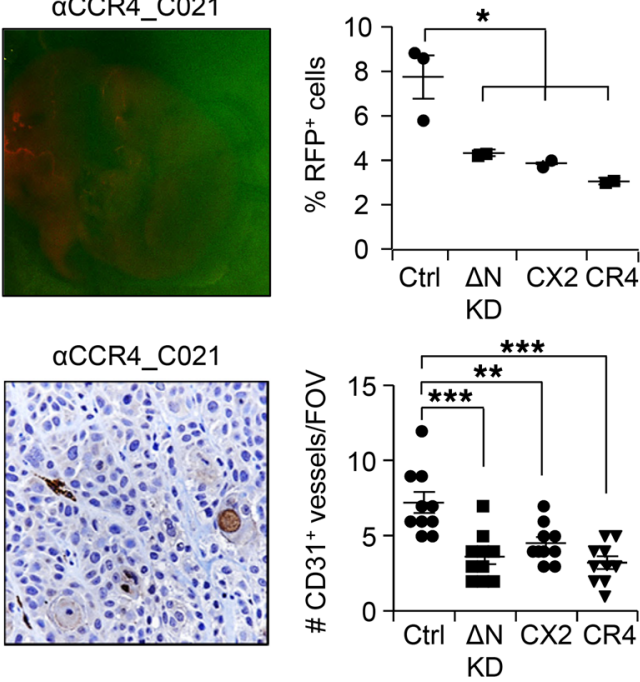

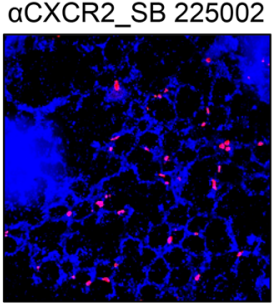

aCCR4_C021
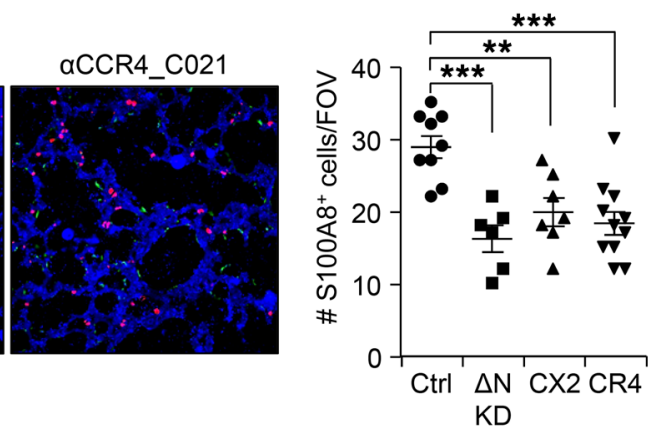

Figure 6. Blockade of MDSC recruitment inhibits metastasis. (A, left) Representative lung images of tumor-bearing mice show RFP ${ }^{+}$nodules (red arrows) upon indicated treatments. HCC1806 tumor cells $\left(2 \times 10^{5}\right)$ were injected into mice. Treatment of mice was started 2 days before injection of tumor cells. $(\mathbf{A}$, right) Flow analysis shows the quantification of metastasized RFP+ tumor cells to the lung. (B) Representative IHC images of primary tumor show CD31+ blood vessels in treated and untreated HCC1806 tumors; quantification is shown at right. (C) HCC1806 tumor cells $\left(2 \times 10^{5}\right)$ were injected into the tail vein of NSG mice. RFP+ metastatic lung nodules upon indicated treatments are shown (red arrows). Quantification of RFP' metastatic lung nodules is shown at right. (D) Representative IF images show the recruitment of S100A8+ cells in the lung upon tail vein injection of HCC1806 cells with indicated treatment (left) and quantification of S100A8 ${ }^{+}$cells (right). Tumor cells are stained with K14 (green). $n$ is indicated in scatter plots. Scale bars: $500 \mu \mathrm{m}$ (A and C) and $80 \mu \mathrm{m}$ (B and $\mathbf{D}) .{ }^{*} P<0.05,{ }^{* *} P<0.01,{ }^{* * *} P<0.001$. (A-D) $P$ values were calculated using 1-way ANOVA with Tukey's multiple-comparisons post hoc test. Data are presented as the mean \pm SEM. (B) $n=3$ samples were used, and several random fields per sample were evaluated for quantification from 3 independent experiments.

both CXCL2 and CCL22, which could be blocked by inhibitors of their cognate receptor (CXCR2 and CCR4), respectively (Figure 5P). Quantitative PCR confirmed higher expression of the chemokine receptors CXCR2 and CCR4 in MDSCs compared with tumor cells (Figure $5 \mathrm{Q}$ ), suggesting paracrine signaling between MDSCs and $\triangle$ Np $63^{+}$TNBC cells. SB 225002 and C021 (Tocris) are well-established CXCR2 and CCR4 inhibitors $(44,45)$. Collectively, our data indicate that the $\triangle$ Np63-dependent chemokines CXCL2 and CCL22 are responsible for recruitment of MDSCs in TNBC.
Blockade of MDSCs by chemokine receptor inhibitors reduces recruitment of MDSCs and reduces metastasis in TNBC. To test the functional significance of increased MDSCs in TNBC, we blocked MDSCs in vivo using 2 independent experimental strategies. First, we performed mammary fat pad injection of HCC1806 TNBC cells in the presence and absence of well-established CXCR2 (SB 225002, Tocris) and CCR4 inhibitors (C021, Tocris) (Supplemental Figure 12A). While there was no significant decrease in tumor initiation and growth of treated HCC1806 tumors compared with 
A

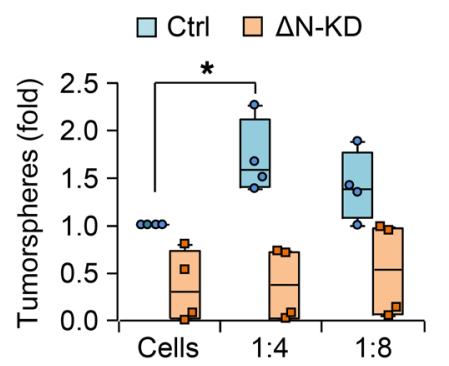

E

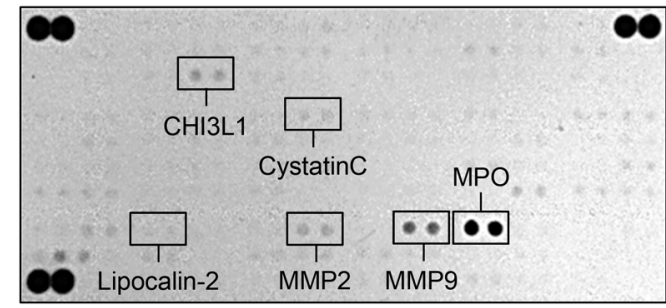

B

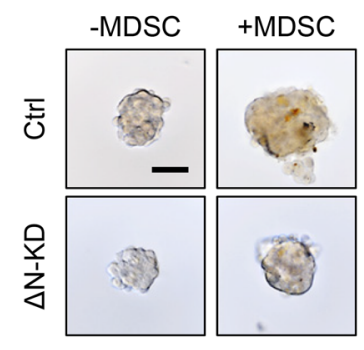

C

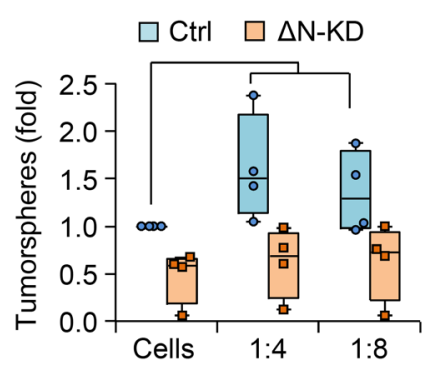

D

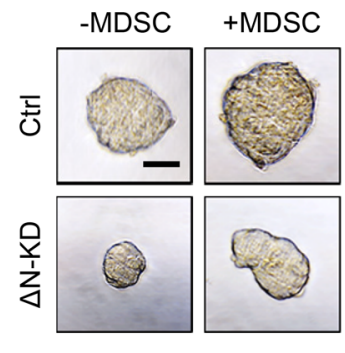

$\mathbf{F}$

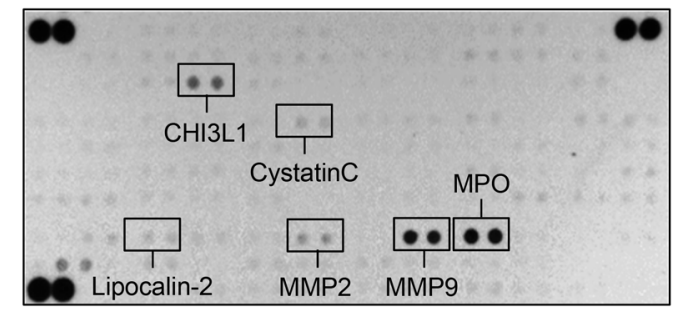

\begin{tabular}{|c|}
\hline Cytokines \\
(MDSC/Neut.) \\
\hline Chitinase3-like 1 \\
MMP2 \\
MMP9 \\
MPO \\
Cystatin C \\
Lipocalin-2 \\
\hline
\end{tabular}

G

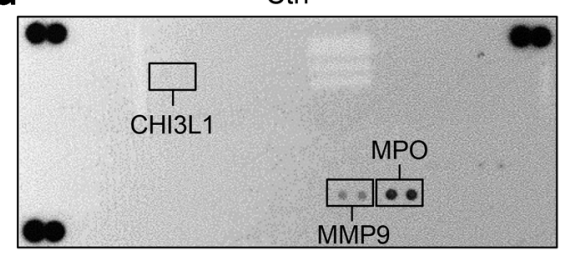

CXCL2

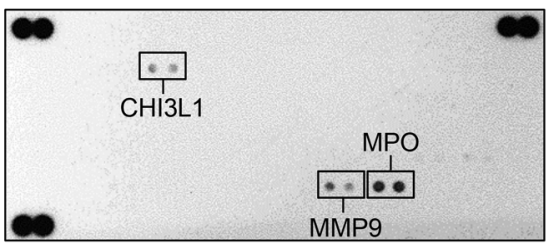

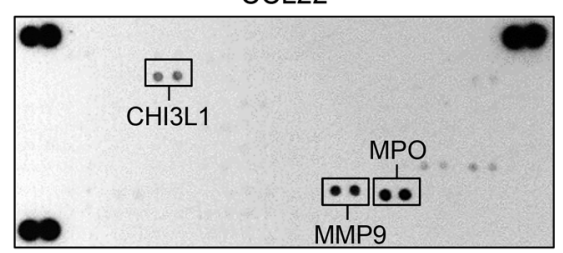

CCL22

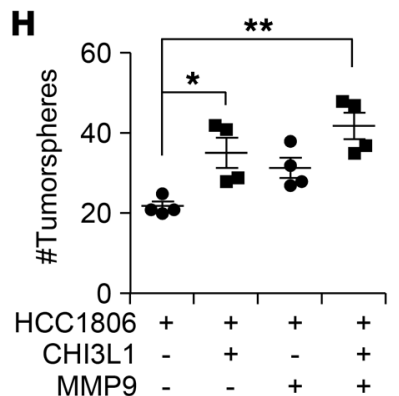

I
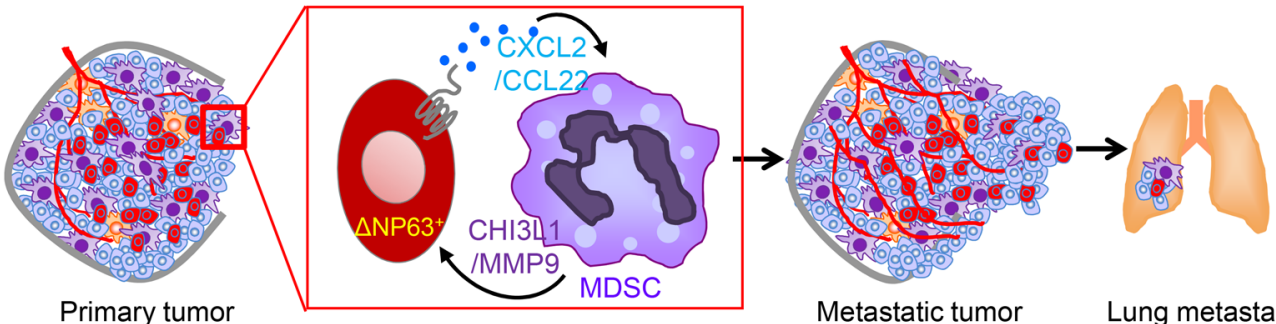

Metastatic tumo

Lung metastasis

Figure 7. MDSCs secrete prometastatic factors and enhance CSC activities of TNBC. (A and B) Number (A) and representative images (B) of tumorspheres from HCC1806 cells cultured for 3 days with or without MDSCs. (C and D) Number (C) and representative images (D) of tumorspheres from SUM159 cells cultured for 3 days with or without MDSCs. (A-D) $n=3$ independent experiments performed in technical duplicate. (E) Representative images of cytokine arrays from cell lysates of normal mammary neutrophils (from mammary gland) and MDSCs from primary mammary tumor. (F) Table shows the most differentially expressed proteins. (C) Representative array blots show differential protein expression upon treatment of MDSCs with recombinant CXCL2 and CCL22 for 12 hours. (H) Scatter plot shows number of tumorspheres from HCC1806 cells upon indicated treatments. Scatter plot show data from 3 independent experiments, and data are presented as the mean \pm SEM. (I) Model shows the recruitment of PMN-MDSCs at primary tumor and at metastatic organ via $\triangle N$ N63 ${ }^{+}$ cancer cells through chemokines. Concomitantly, MDSCs secrete factors such as chitinase 3-like 1 (CHI3L1) and MMPg to enhance both tumor growth and metastatic potential. Scale bars: $40 \mu \mathrm{m}$ (B and $\mathbf{D}$ ). Data are presented as the mean $\pm \mathrm{SEM}$. ${ }^{*} P<0.05 ;{ }^{*} P<0.01$. $P$ value was calculated using 1 -way ANOVA with Tukey's multiple-comparisons post hoc test.

untreated control tumors (Supplemental Figure 12, B and C), there was a significant decrease in $\mathrm{Ly}_{6 \mathrm{G}}{ }^{+} \mathrm{MDSC}$ recruitment to the primary tumor site (Supplemental Figure 12, D and E) and a significant reduction in the number of lung metastatic lesions in treated mice compared with controls (Figure 6A). The total percentage of Ly6G+ PMN-MDSCs in blood increased upon inhibitor treatment (Supplemental Figure 12, F and G), suggesting that in the absence of CXCR2 and CCR4 signaling, MDSCs lose their ability to migrate to metastatic sites. IHC on treated primary tumors showed no difference in the number of either Ki $67^{+}$proliferating cells (Supplemental Figure 12H) or cleaved caspase- $3^{+}$apoptotic cells compared with control (data not shown), in agreement with our finding that primary tumor growth was not significantly affected by treatment. However, the number of CD $31^{+}$blood vessels was significantly reduced by treatment (Figure 6B), supporting the well-established function of MDSCs in angiogenesis $(46,47)$. In parallel, tail vein injection studies using control HCC1806 along with CXCR2 and CCR4 inhibitors showed significantly fewer met- 
astatic events at multiple distal organs, including lung, compared with control, phenocopying the $\triangle$ Np63-KD HCC1806 cells (Figure $6 \mathrm{C}$ and Supplemental Figure 13, A-D). As expected, recruitment of MDSCs (as seen by reduced $\mathrm{S} 100 \mathrm{~A} 8^{+}$cells) was greatly reduced by either CXCR2 or CCR4 inhibitors or by reduced levels of $\Delta \mathrm{Np} 63$ (Figure 6D). Taken together, our data indicate that, as with $\triangle$ Np63-KD HCC1806 cells, blocking CXCR2 and CCR4 prevents MDSC recruitment to tumor sites, leading to reduced angiogenesis and metastasis.

Nonimmunologic function of MDSCs promoting cancer stem cells in TNBC. Although MDSCs suppress immune T and NK cell proliferation $(8,9)$, recent studies show that they may also function in an immune-independent fashion to enhance cancer stem cells (CSCs) in pancreatic and ovarian cancers $(48,49)$. Our xenograft studies in immunocompromised mice strongly (Figures 2 and 3) support a nonimmunologic function for MDSCs, which was further supported by studies using EpRas cells in BALB/c mice (Supplemental Figure 9, G, H, M, and N). To determine whether MDSCs influence CSCs in TNBC, we performed tumorsphere assays, which serve as an in vitro surrogate for tumor-initiating cell (TIC)/CSC function. Cells grown in in vitro tumorspheres expressed higher levels of $\Delta$ Np63 compared with cells in monolayer cultures, suggesting that $\triangle \mathrm{Np} 63$ is enriched in CSCs (Supplemental Figure 14A). Furthermore, we found that $\triangle \mathrm{Np} 63$ enhanced CSC activity as the number of generated tumorspheres was reduced in $\triangle$ Np63-KD HCC1806 cells compared with control (Figure 7, A and B), supporting our previously published studies. Testing the tumor-promoting function of MDSCs on CSCs in vitro, we found a significant increase in the number of tumorspheres when control HCC1806 cells were cocultured with tumor MDSCs (Figure 7, A and B). In contrast, $\triangle$ Np63-KD HCC1806 cells showed no difference in tumorsphere number when MDSCs were added, indicating that the MDSC effect depended on tumor-specific $\Delta \mathrm{Np} 63$ expression (Figure 7, $A$ and B). This phenotype was further confirmed in SUM159 (Figure 7, C and D), suggesting that MDSCs promote CSC function in TNBC in a $\triangle$ Np63-dependent manner.

To determine whether the interaction between MDSCs and

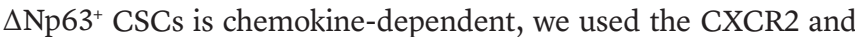
CCR4 inhibitors. Notably, inclusion of these inhibitors decreased the number and size of tumorspheres in both of the TNBC cell lines (Supplemental Figure 14, B-E), suggesting a potential chemokine-dependent feedback mechanism promoting MDSC-driven tumorigenic activity of $\triangle \mathrm{Np} 63^{+} \mathrm{CSC}$ in TNBCs. To identify any MDSC-secreted factors that might promote this activity, we undertook a comparative cytokine array analysis of normal mammary neutrophils and MDSCs from mammary tumors (HCC1806 and SUM159 cells). Of 111 cytokines/secreted factors tested, 6 were significantly different (Figure 7, E and F), and the 2 secreted factors that showed the highest differential expression were CHI3L1 and MMP9 (Figure 7, E and F). Remarkably, CXCL2/CCL22 ligand treatment of MDSCs for 12 hours specifically upregulated the expression of CHI3L1 and MMP9 proteins (out of 111 proteins) (Figure 7G), which was further confirmed with supernatant from control and $\Delta \mathrm{Np} 63$ KD TNBC cells in culture (data not shown), indicating a direct effect of $\Delta$ Np63-targeted chemokines on MDSC activity. CHI3L1 and MMP9 have a strong metastasis-promoting function in cancer, including breast cancer (50-52). However, secretion of these factors by MDSCs and their function in TNBCs had not yet been explored. Here, we find that addition of CHI3L1 and MMP9 lead to a dramatic increase in the number of tumorspheres, demonstrating the CSC-promoting function of these prometastatic secreted factors in TNBCs (Figure 7H). These data indicate a novel nonimmunologic function of MDSC-expressed CHI3L1 and MMP9 in promoting the $\triangle \mathrm{Np} 3^{+} \mathrm{CSC}$ activity of TNBCs.

\section{Discussion}

The plasticity and heterogeneity of TNBC cells make them difficult to target therapeutically. However, as the TME plays an important role in dictating the fate of cancer cells $(5-7,53)$, enthusiasm for therapeutically targeting this population in combination with antitumor therapy is increasing. Immune cells within the TME play an essential role in tumor progression and metastasis. While increased numbers of MDSCs in circulating blood correlate with tumor burden, stage of tumor, and metastasis in breast cancer patients, their representation within the TME and the potential role of tumor-associated MDSCs in driving the aggressive nature and metastasis of TNBC remained unknown. In this study, for the first time to our knowledge, we showed that PMN-MDSCs are increased in the more aggressive TNBC subset compared with the non-TNBC subset. Furthermore, our data showed that more PMN-MDSCs are present in basal-like TNBC, the most aggressive subset of TNBC $(28,29)$, which also has increased expression of $\Delta$ Np63 protein. Moreover, high levels of p63 (predominantly the $\Delta$ Np63 isoform) (21) correlate with reduced metastasis-free survival in TNBC patients. Our functional data demonstrated that $\triangle$ Np63 promotes tumor progression and metastasis by recruiting MDSCs to the TME of both human and mouse TNBCs. This effect is TNBC-specific, as overexpression of $\triangle N p 63$ in non-TNBCs was unable to promote tumor growth or angiogenesis. However, the fate of basal $\mathrm{K}^{+} 4^{+}$cells in non-TNBCs was altered when $\Delta \mathrm{Np} 63$ was overexpressed, corroborating earlier data that $\Delta \mathrm{Np} 63$ is a major driver of basal cell fate (21). MDSCs contribute to metastases by aiding in the formation of the premetastatic niche and promoting angiogenesis and tumor cell invasion $(47,54)$. Our data support this notion and demonstrate that loss of $\triangle \mathrm{Np} 63$ in TNBCs is associated with reduced angiogenesis, marked by reduced vessel density and increased vessel integrity. Finally, the metastasis-promoting function of $\triangle \mathrm{Np} 63$ in TNBC was confirmed in both mammary fat pad and experimental tail vein injection assays that bypass the primary tumor growth effect. Overall, our study clearly established that $\triangle \mathrm{Np} 63$ promotes TNBC tumor growth, progression, angiogenesis, and metastasis, including extravasation to peripheral blood and colonization in distant metastatic organs.

Mechanistically, our studies demonstrate that $\Delta \mathrm{Np} 63$ promotes MDSC recruitment to primary and metastatic sites by transcriptionally activating chemokines such as CXCL2 and CCL22. Notably, CXCL2 and CCL22 expression positively correlates with TP63 and is associated with poor DMFS in TNBC patients, supporting the clinical relevance of our findings. Interestingly, CXCL2/ CCL22 was associated with improved DMFS in non-TNBC patients, suggesting a TNBC-specific function of $\triangle$ Np63/CXCL2/ CCL22 signaling in MDSC recruitment and metastasis. Indeed, functional studies demonstrate that blocking CXCR2/CCR4 inhibits angiogenesis and metastasis in TNBC, phenocopying 
knockdown of $\Delta$ Np63. However, we found that inhibition of CXCR2/CCR4 does not inhibit primary tumor growth despite reducing MDSCs, suggesting the requirement for additional downstream targets of $\triangle \mathrm{Np} 63$ in TNBC tumor growth. It is also possible that the number of tumor cells at injection time was higher, minimizing the effect of CXCR2/CCR4 inhibitors on tumor volume, which needs further evaluation.

$\triangle$ Np63 facilitates the recruitment of MDSCs and promotes metastasis of human and mouse TNBC cells in both immune-competent and immune-compromised models, highlighting a nonimmunologic function for MDSCs in the TME of TNBCs that is independent of their role in T cell and NK cell suppression. This view is supported by recent studies suggesting that MDSCs promote metastasis of pancreatic and ovarian cancer by enhancing CSC-like properties $(48,49)$. Indeed, our data with mouse TNBC cells show that loss of $\triangle \mathrm{Np} 63$ at primary and metastatic sites reduces tumor growth, progression, and metastasis by reducing recruitment of MDSCs. Supporting prior studies that show that MDSCs enhance CSC activity of breast cancer cells through cell-cell contact signaling $(55,56)$, we find that MDSCs increase CSC activity in a $\triangle$ Np63-dependent manner in TNBCs. We find that greater numbers of MDSCs are present in the TME of TNBCs than in other subtypes. Moreover, we find an intriguing link where MDSCs promote CSC function in TNBC through secreted factors that include MMP9 or CHI3L1. Notably, addition of either MMP9 or CHI3L1 enhances tumorsphere numbers, indicative of their direct effects on CSCs. MMP9 is important for metastasis of TNBC cancer cells, and high levels of MMP9 in tumor cells are associated with poor prognosis of TNBC patients $(50-52,57)$. Although MMP9 has been implicated in the mobility of MDSCs during hematopoiesis (58), its function within the context of the TME of TNBCs has not been established. Similarly, while CHI3L1 is important for pulmonary metastasis, the potential impact of MDSC-expressed CHI3L1 is completely unknown. The high rate of tumor relapse, metastasis, and therapeutic resistance of TNBC is often linked to increased numbers of $\operatorname{CSCs}(59,60)$. Our data suggest that prometastatic factors such as MMP9 and CHI3L1 secreted from MDSCs promote $\triangle \mathrm{Np} 63^{+} \mathrm{CSCs}$, which then in turn facilitate increased metastasis of TNBC cells (Figure 7I).

In summary, our study sheds light on a novel crosstalk between MDSCs and $\triangle$ Np63-enriched CSCs in promoting TNBC metastasis (Figure 7I). These findings provide a rationale for developing CXCR2 and CCR4 antagonists and neutralizing antibodies as therapeutic approaches for $\triangle \mathrm{Np} 63^{\text {high }} \mathrm{TNBC}$ patients. Our data further highlight the potential use of $\triangle \mathrm{Np} 63$ as a prognosis marker to stratify TNBC patients and suggest that basal TNBC patients with high $\triangle \mathrm{Np} 63$ will specifically benefit from adjunct CXCR2/ CCR4-targeted therapy.

\section{Methods}

Human patient samples. Breast cancer specimens used in the study were deidentified samples and were obtained from the Eastern Division of the Cooperative Human Tissue Network, University of Pennsylvania. All antibodies were incubated at $4^{\circ} \mathrm{C}$ for 12 hours. All samples were scored for both intensity of protein and abundance of positive cells when IHC was performed. The intensity of the protein was measured using a $0-3$ scale, 0 being negative and 3 being very high expression. The abundance of positive cells in the tissue was measured using a scale ranging from 0 to 100 . To score abundance, a score of more than 1 was considered positive. $\mathrm{H}$-score $=$ intensity $\times$ abundance. For immunofluorescence studies, only abundance was measured. The stromal cells were scored for CD11B, CD33/S100A9, and CD15/Lox1 markers. The tumor cells were scored for $\triangle \mathrm{Np} 63$ and K14 markers. For $\Delta \mathrm{Np} 63$, nuclear staining was considered. Mann-Whitney $U$ test was used for box plots in Figure 1, D and E, and Supplemental Figure 1, $\mathrm{F}-\mathrm{H}$. The scoring of specimen slides was done in a blinded manner. The researcher or pathologist had no prior knowledge of the slide identity.

Animal studies. BALB/c, NSG, FVB, and athymic nude mice were obtained from The Jackson Laboratory. For mammary fat pad injection experiments, all mice at 5-7 weeks old were anesthetized, and tumor cells were injected into the mammary fat pad following established protocols (61). All mammary fat tumors were palpated every week. The experiment was terminated when tumors reached $1,500 \mathrm{~mm}^{2}$ in size, or was terminated earlier if the tumors were ulcerated (humane endpoint). For i.v. injection, the tumor cells were injected into the tail vein of 5- to 6-week-old mice following established protocol (62).

Cell culture studies. The HCC1806 cell line was obtained from Sophie Ran (Southern Illinois University, Carbondale, Illinois, USA), whereas the SUM159, MCF7, EpRas, and WTB cell lines were obtained from Yibin Kang (Princeton University, Princeton, New Jersey, USA). HCC1806, MCF7, EpRas, and WTB cell lines were grown in DMEM (Sigma-Aldrich), and the SUM159 cell line was grown in F12 medium, supplemented with $10 \%$ FBS (Invitrogen), $100 \mathrm{U} / \mathrm{ml}$ penicillin, and $100 \mu \mathrm{g} / \mathrm{ml}$ streptomycin sulfate (Invitrogen). Cells were tested and confirmed to be mycoplasma-negative. Human breast cancer cells were authenticated by short tandem repeat DNA profiling analysis.

Viral production and infection. For lentiviral-mediated knockdown studies, we used one of the previously validated isoform-specific shRNAs for human and mouse $\triangle$ Np63 shRNAs, respectively (21). The targeting shRNA sequence for mouse $\triangle \mathrm{Np} 63$ is $5^{\prime}$-GGCACCTGAATTCTGTTA-3' for $\triangle N p 63-K D$. The targeting shRNA sequence for human $\triangle$ Np63-KD is 5'-GCAGCATTGATCAATCTTA-3'. We used previously validated expression plasmid for $\triangle \mathrm{Np} 63$ overexpression studies (21). Viral packaging followed by subsequent infection of cells was done following standard protocols (21). For validated knockdown constructs (21), $\mathrm{GFP}^{+}$cells were enriched using flow cytometry since cells were labeled with GFP. $\triangle$ Np63-overexpressing WTB cells were selected with puromycin.

Protein extraction and Western blot analysis. Proteins were extracted from cell lines in RIPA buffer as previously described (63). Western blot analysis was performed using the standard protocol. Antibodies and dilutions used are listed in Supplemental Table 1. See complete unedited blots in Supplemental Figure 15.

Histological analysis, IHC, and immunofluorescence. For histological analysis, human patient samples and mouse tumor and mouse lung specimens were processed as previously described (62). Antibody dilutions are listed in Supplemental Table 1. For IHC, antibody slides were counterstained with hematoxylin. For immunofluorescence, DAPI was used to stain nuclei. Images were taken with a Nikon TiE microscope.

Flow cytometry/FACS sorting. The single-cell suspension for flow cytometry analysis of tumor, lung, or spleen was obtained following published protocol (21). Single cells were stained with a combination of antibodies (listed in Supplemental Table 2) for 20-30 minutes at room temperature in the dark. FACS analysis was performed using 
the LSRII Flow Cytometer (BD Biosciences), and data were analyzed using FlowJo software (Tree Star Inc.). For sorting of cells, Aria II or FACS Jazz instruments were used. Antibodies and dilutions used are listed in Supplemental Table 2.

$R N A$ and quantitative reverse transcriptase PCR analysis. Total RNA was isolated from cultured cell lines, primary cells, and sorted cells using standard method. The gene-specific primer sets were used at a final concentration of $0.2 \mu \mathrm{M}$, and their sequences are listed in Supplemental Table 3.

Chromatin immunoprecipitation. HCC1806 cells were grown to $80 \%$ confluence, and cells were then cross-linked with $1 \%$ formaldehyde and processed. ChIP-Seq experiments were performed with 2 independent p63 antibodies using the iDeal ChIP-Seq kit for Transcription Factors (Diagenode) (64).

RNA-Seq analysis and gene set enrichment analysis. RNA-Seq was performed by DNA Link Inc. (California, USA) according to the manufacturer's protocol from Kapa Biosystems (RNA-seq Library Quantification Kit). Thirty million reads were obtained from each sample. Data were deposited in the NCBI's Gene Expression Omnibus (GEO) database with accession number GSE102377. For gene expression analysis, reads were aligned to the hg38 UCSC assembly using TopHat version 2.0.14 and Bowtie version 2.10. GFF files were obtained from Genome Reference Consortium Human Build 38 patch release 9. The distribution of alignments was analyzed using Cufflinks version 2.2.1. FPKM values were normalized. Differential expression testing was performed using Cuffdiff version 2.2.1; however, all FPKM values are those calculated by Cufflinks.

Gene set enrichment analysis (GSEA) was conducted using GSEA2.2.4 software to generate enrichment scores for gene sets in Hallmark C2.all, C5.all, and C6.all data sets with default settings. Gene sets were tested for enrichment in rank-ordered lists via GSEA using a weighted Kolmogorov-Smirnov-like statistic to calculate the enrichment score. The RNA-Seq data were deposited in the GEO database with accession number GSE102377.

Protein arrays and ELISA. MDSCs from the HCC1806 and SUM159 xenograft tumors were isolated to perform protein array. In brief, xenograft tumors were enzymatically digested to get cells in suspension as described previously (62). Neutrophils from normal breast or MDSCs were enriched using anti-Gr1 particles via magnetic separation (catalog 558111, BD Biosciences). Enriched MDSCs were stained with a combination of antibody cocktail for 30 minutes in dark at room temperature. MDSCs $\left(\mathrm{CD} 45^{+} \mathrm{CD} 11 \mathrm{~B}^{+} \mathrm{Gr} 1^{+}\right)$ were isolated using a flow cytometry sorter. Chemokine array (R\&D Systems) and ELISA (R\&D Systems) were performed according to the manufacturer's instructions. Conditioned medium from control and $\Delta$ Np63-KD cells was used to determine the levels of chemokines. Cytokine array (R\&D Systems) was performed according to the manufacturer's instructions. Sorted cell lysates from neutrophils and MDSCs were used to determine the levels of cytokines/secreted factors. For ELISA, HCC1806 and SUM159 cells were cultured into a $10-\mathrm{cm}$ dish. After overnight incubation, the level of CXCL2 chemokine was evaluated by ELISA.

$T$ cell proliferation and suppression assay. $\mathrm{T}$ cell proliferation and suppression assays were performed following protocols in published literature (56). IFN- $\gamma$ staining was examined via flow cytometry after 3-4 days. Samples were also stained for Gr1, to exclude MDSCs from analysis.
In vivo treatment experiments. For drug treatments, mice were randomly assigned to the cohorts. Mice were treated with CXCR2 antagonist (catalog 2725, R\&D Systems) or CCR4 antagonist (catalog 3581 , R\&D Systems) at $2 \mathrm{mg} / \mathrm{kg}$ on alternating days. See Supplemental Table 4 for details.

Bioluminescence imaging of tumor and lung tissues. Mice were anesthetized and injected with $100 \mu \mathrm{l}$ of Renilla luciferin substrate (30 $\mathrm{mg} / \mathrm{ml}$; Gold Biotechnology). Bioluminescence images were obtained using the IVIS Spectrum system (Caliper Life Sciences). Data are expressed as total photon flux and were analyzed using Living Image 3.0 software (Caliper Life Sciences).

CTC culture and quantification. The same amount of peripheral blood was isolated from xenograft tumor-bearing mice of different groups and processed for RBC lysis followed by culture in tissue culture dishes for 5-7 days or extraction of total genomic DNA using a Qiagen DNA extraction kit following the manufacturer's protocol. Semiquantitative PCR was performed with human and mouse genomic DNA using GAPDH primers (Supplemental Table 3). The intensities of PCR-electrophoresis gel bands were quantified using Image J software (NIH), and the values were subtracted from background values. Semiquantitative data from human GAPDH were normalized with mouse GAPDH using genomic DNA to quantify the levels of human CTCs in mouse blood.

Tumorsphere assay. Tumor cells were cultured as previously described (62). For coculture tumorsphere assay with TNBC cells and MDSCs, both cell types were mixed in a 1:4 or 1:8 ratio and grown in Corning low-adherent plates in tumorsphere media for 3-5 days (62). All recombinant chemokines and growth modulators (R\&D Systems) (listed in Supplemental Table 4) were used in $100 \mathrm{ng} / \mathrm{ml}$ concentration.

Migration assay. Mouse primary MDSCs were allowed to migrate through Costar Transwell permeable 8 - $\mu \mathrm{m}$-pore-size membranes in 24-well plates (Corning) toward the conditioned medium of TNBC cells or chemokines such as CXCL2 or CCL22 (100 ng/ml) for 1-3 hours at $37^{\circ} \mathrm{C}$. Total migrated MDSCs were counted by collection of all MDSCs at the bottom of the 24-well plate. All recombinant mouse chemokines (R\&D Systems; listed in Supplemental Table 4) were used in $100 \mathrm{ng} / \mathrm{ml}$ concentration. CXCR2 and CCR4 synthetic blocking compounds (R\&D Systems; Supplemental Table 4) were used at 2 $\mu \mathrm{g} / \mathrm{ml}$ and $5 \mu \mathrm{g} / \mathrm{ml}$ concentration, respectively.

Statistics. Results were reported as mean \pm SD or mean \pm SEM. The significance of differences was calculated using 2-tailed Student's $t$ test for normally distributed data sets. Normal distribution of data was evaluated by Shapiro-Wilk $W$ test or Skewness-Kurtosis test for normality. Non-normally distributed data sets were analyzed using nonparametric Mann-Whitney $U$ tests. The tumor growth data sets were analyzed using the Bonferroni corrected 2-way ANOVA to compute statistical significance. One-way ANOVA with Tukey's post hoc test was done for samples with multiple comparisons. For TNBC and non-TNBC KM plots (Figure 1, F-H), the KM Plotter "auto select best cutoff" option was used for stratification. Differences in survival between groups via Kaplan-Meier plots were statistically evaluated using log-rank tests. Mann-Whitney $U$ test was used for correlation scatter plots using human patient samples. A $P$ value of less than 0.05 was considered statistically significant.

Study approval. Breast cancer specimens used in the study were deidentified samples and were obtained from the Eastern Division of the Cooperative Human Tissue Network, University of Pennsylvania. All samples were considered exempt by the Institutional Review Board of the University of Pennsylvania. Animal procedures were conducted 
in compliance with and with the approval of the Institutional Animal Care and Use Committee of the University of Pennsylvania.

\section{Author contributions}

SK and RC designed all experiments. SK, DWW, and NS performed all the experiments. AN provided technical advice and performed the scoring on human breast cancer samples after IHC. MAB performed all the clinical and statistical analyses using human breast cancer samples and data sets. KAT and DG provided experimental support in MDSC-related experiments. CG and SS performed and analyzed ChIP-Seq analysis. SK and RC wrote the manuscript. All authors discussed the results and commented on the manuscript.

\section{Acknowledgments}

We thank Serge Y. Fuchs, Ellen Puré, Oliver A. Garden, Manti Guha, and Leslie King (University of Pennsylvania) for critical reading of the manuscript and helpful discussions. We thank the Penn Vet Comparative Pathology Core for their assistance with embedding and sectioning of tumor samples. We thank the Eastern Division of the Cooperative Human Tissue Network, University of Pennsylvania, for providing human breast cancer fixed tissues from patients. We thank the members of Flow Cytometry Core at the Children's Hospital of Philadelphia and the University of Pennsylvania. This work was supported by grants from the American Cancer Society and the McCabe Fund Fellow Award from the University of Pennsylvania (to RC) as well as an NCI K22 grant (K22CA193661 to RC).

Address correspondence to: Rumela Chakrabarti, Department of Biomedical Sciences, School of Veterinary Medicine, University of Pennsylvania, 380 S. University Avenue, 411 Hill Pavilion, Philadelphia, Pennsylvania 19104, USA. Phone: 215.746.1873; Email: rumela@vet.upenn.edu.
1. Dai $X$, et al. Breast cancer intrinsic subtype classification, clinical use and future trends. Am J Cancer Res. 2015;5(10):2929-2943.

2. Bianchini G, Balko JM, Mayer IA, Sanders ME, Gianni L. Triple-negative breast cancer: challenges and opportunities of a heterogeneous disease. Nat Rev Clin Oncol. 2016;13(11):674-690.

3. Gluz O, Liedtke C, Gottschalk N, Pusztai L, Nitz U, Harbeck N. Triple-negative breast cancercurrent status and future directions. Ann Oncol. 2009;20(12):1913-1927.

4. Lehmann BD, et al. Identification of human triple-negative breast cancer subtypes and preclinical models for selection of targeted therapies. JClin Invest. 2011;121(7):2750-2767.

5. Hanahan D, Coussens LM. Accessories to the crime: functions of cells recruited to the tumor microenvironment. Cancer Cell. 2012;21(3):309-322.

6. Fessler E, Dijkgraaf FE, De Sousa E Melo F, Medema JP. Cancer stem cell dynamics in tumor progression and metastasis: is the microenvironment to blame? Cancer Lett. 2013;341(1):97-104.

7. Quail DF, Joyce JA. Microenvironmental regulation of tumor progression and metastasis. Nat Med. 2013;19(11):1423-1437.

8. Gabrilovich DI. Myeloid-derived suppressor cells. Cancer Immunol Res. 2017;5(1):3-8.

9. Marvel D, Gabrilovich DI. Myeloid-derived suppressor cells in the tumor microenvironment: expect the unexpected. JClin Invest. 2015;125(9):3356-3364.

10. Gabrilovich DI, Nagaraj S. Myeloid-derived suppressor cells as regulators of the immune system. Nat Rev Immunol. 2009;9(3):162-174.

11. Bronte V. Myeloid-derived suppressor cells in inflammation: uncovering cell subsets with enhanced immunosuppressive functions. Eur $J$ Immunol. 2009;39(10):2670-2672.

12. Mills AA. p63: oncogene or tumor suppressor? Curr Opin Genet Dev. 2006;16(1):38-44.

13. Crum CP, McKeon FD. p63 in epithelial survival, germ cell surveillance, and neoplasia. Annu Rev Pathol. 2010;5:349-371.

14. Yang A, et al. p63, a p53 homolog at 3q27-29, encodes multiple products with transactivating, death-inducing, and dominant-negative activi- ties. Mol Cell. 1998;2(3):305-316.

15. Romano RA, et al. $\Delta$ Np63 knockout mice reveal its indispensable role as a master regulator of epithelial development and differentiation. Development. 2012;139(4):772-782.

16. Su X, et al. TAp63 suppresses metastasis through coordinate regulation of Dicer and miRNAs. Nature. 2010;467(7318):986-990.

17. Westfall MD, Pietenpol JA. p63: Molecular complexity in development and cancer. Carcinogenesis. 2004;25(6):857-864.

18. Saladi SV, et al. ACTL6A is co-amplified with p63 in squamous cell carcinoma to drive YAP activation, regenerative proliferation, and poor prognosis. Cancer Cell. 2017;31(1):35-49.

19. Sinha A, Chandra S, Raj V, Zaidi I, Saxena S, Dwivedi R. Expression of p63 in potentially malignant and malignant oral lesions. JOral Biol Craniofac Res. 2015;5(3):165-172.

20. Keyes WM, et al. $\Delta \mathrm{Np} 63 \alpha$ is an oncogene that targets chromatin remodeler Lsh to drive skin stem cell proliferation and tumorigenesis. Cell Stem Cell. 2011;8(2):164-176.

21. Chakrabarti R, et al. $\Delta$ Np63 promotes stem cell activity in mammary gland development and basal-like breast cancer by enhancing Fzd7 expression and Wnt signalling. Nat Cell Biol. 2014;16(10):1004-1015.

22. Memmi EM, et al. p63 Sustains self-renewal of mammary cancer stem cells through regulation of Sonic Hedgehog signaling. Proc Natl Acad Sci US A. 2015;112(11):3499-3504.

23. Du Z, et al. Overexpression of $\Delta \mathrm{Np} 63 \alpha$ induces a stem cell phenotype in MCF7 breast carcinoma cell line through the Notch pathway. Cancer Sci. 2010;101(11):2417-2424.

24. Bronte V, et al. Recommendations for myeloid-derived suppressor cell nomenclature and characterization standards. Nat Commun. 2016;7:12150.

25. Condamine T, et al. Lectin-type oxidized LDL receptor-1 distinguishes population of human polymorphonuclear myeloid-derived suppressor cells in cancer patients. Sci Immunol. 2016;1(2):aaf8943.

26. Nekulova M, et al. $\Delta$ Np63 $\alpha$ expression induces loss of cell adhesion in triple-negative breast cancer cells. BMC Cancer. 2016;16(1):782.

27. Lodillinsky C, et al.p63/MT1-MMP axis is required for in situ to invasive transition in basal-like breast cancer. Oncogene. 2016;35(3):344-357.

28. Rakha EA, et al. Triple-negative breast cancer: distinguishing between basal and nonbasal subtypes. Clin Cancer Res. 2009;15(7):2302-2310.

29. Jacquemier J, et al. Protein expression profiling identifies subclasses of breast cancer and predicts prognosis. Cancer Res. 2005;65(3):767-779.

30. Györffy B, et al. An online survival analysis tool to rapidly assess the effect of 22,277 genes on breast cancer prognosis using microarray data of 1,809 patients. Breast Cancer Res Treat. 2010;123(3):725-731.

31. Pereira B, et al. The somatic mutation profiles of 2,433 breast cancers refines their genomic and transcriptomic landscapes. Nat Commun. 2016;7:11479.

32. Curtis C, et al. The genomic and transcriptomic architecture of 2,000 breast tumours reveals novel subgroups. Nature. 2012;486(7403):346-352.

33. Volk-Draper LD, Rajput S, Hall KL, Wilber A, Ran S. Novel model for basaloid triple-negative breast cancer: behavior in vivo and response to therapy. Neoplasia. 2012;14(10):926-942.

34. Suarez-Carmona $\mathrm{M}$, et al. $\Delta \mathrm{Np} 63$ isoform-mediated $\beta$-defensin family up-regulation is associated with (lymph)angiogenesis and poor prognosis in patients with squamous cell carcinoma. Oncotarget. 2014;5(7):1856-1868.

35 . Bid HK, et al. $\Delta$ Np63 promotes pediatric neuroblastoma and osteosarcoma by regulating tumor angiogenesis. Cancer Res. 2014;74(1):320-329.

36. Folkman J. Role of angiogenesis in tumor growth and metastasis. Semin Oncol. 2002;29(6 suppl 16):15-18.

37. Wang WQ, et al. Intratumoral $\alpha$-SMA enhances the prognostic potency of CD34 associated with maintenance of microvessel integrity in hepatocellular carcinoma and pancreatic cancer. PLOS One. 2013;8(8):e71189.

38. Janda E, Litos G, Grünert S, Downward J, Beug H. Oncogenic Ras/Her-2 mediate hyperproliferation of polarized epithelial cells in $3 \mathrm{D}$ cultures 
and rapid tumor growth via the PI3K pathway. Oncogene. 2002;21(33):5148-5159.

39. Fagiani E, Christofori G. Angiopoietins in angiogenesis. Cancer Lett. 2013;328(1):18-26.

40. Wan L, et al. MTDH-SND1 interaction is crucial for expansion and activity of tumor-initiating cells in diverse oncogene- and carcinogen-induced mammary tumors. Cancer Cell. 2014;26(1):92-105.

41. Herschkowitz JI, et al. Identification of conserved gene expression features between murine mammary carcinoma models and human breast tumors. Genome Biol. 2007;8(5):R76.

42. Palacios-Arreola MI, Nava-Castro KE, Castro JI, García-Zepeda E, Carrero JC, Morales-Montor J. The role of chemokines in breast cancer pathology and its possible use as therapeutic targets. JImmunol Res. 2014;2014:849720.

43. Sarvaiya PJ, Guo D, Ulasov I, Gabikian P, Lesniak MS. Chemokines in tumor progression and metastasis. Oncotarget. 2013;4(12):2171-2185.

44. Bento AF, Leite DF, Claudino RF, Hara DB, Leal PC, Calixto JB. The selective nonpeptide CXCR2 antagonist SB225002 ameliorates acute experimental colitis in mice. J Leukoc Biol. 2008;84(4):1213-1221.

45. McMillin M, et al. Neuronal CCL2 is upregulated during hepatic encephalopathy and contributes to microglia activation and neurological decline. J Neuroinflammation. 2014;11:121.

46. Umansky V, Blattner C, Gebhardt C, Utikal J. The role of myeloid-derived suppressor cells (MDSC) in cancer progression. Vaccines (Basel). 2016;4(4):E36.

47. Binsfeld M, et al. Granulocytic myeloid-derived suppressor cells promote angiogenesis in the context of multiple myeloma. Oncotarget. 2016;7(25):37931-37943.
48. Panni RZ, et al. Tumor-induced STAT3 activation in monocytic myeloid-derived suppressor cells enhances stemness and mesenchymal properties in human pancreatic cancer. Cancer Immunol Immunother. 2014;63(5):513-528.

49. Cui TX, et al. Myeloid-derived suppressor cells enhance stemness of cancer cells by inducing microRNA101 and suppressing the corepressor CtBP2. Immunity. 2013;39(3):611-621.

50. Ma B, et al. RIG-like helicase regulation of chitinase 3-like 1 axis and pulmonary metastasis. Sci Rep. 2016;6:26299.

51. Mehner C, Hockla A, Miller E, Ran S, Radisky DC, Radisky ES. Tumor cell-produced matrix metalloproteinase 9 (MMP-9) drives malignant progression and metastasis of basal-like triple negative breast cancer. Oncotarget. 2014;5(9):2736-2749.

52. Yan $\mathrm{HH}$, et al. Gr-1+CD11 $\mathrm{b}^{+}$myeloid cells tip the balance of immune protection to tumor promotion in the premetastatic lung. Cancer Res. 2010;70(15):6139-6149.

53. Plaks V, Kong N, Werb Z. The cancer stem cell niche: how essential is the niche in regulating stemness of tumor cells? Cell Stem Cell. 2015;16(3):225-238.

54. Condamine T, Ramachandran I, Youn JI, Gabrilovich DI. Regulation of tumor metastasis by myeloid-derived suppressor cells. Annu Rev Med. 2015;66:97-110.

55. Peng D, et al. Myeloid-derived suppressor cells endow stem-like qualities to breast cancer cells through IL6/STAT3 and NO/NOTCH cross-talk signaling. Cancer Res. 2016;76(11):3156-3165.

56. Welte T, et al. Oncogenic mTOR signalling recruits myeloid-derived suppressor cells to promote tumour initiation. Nat Cell Biol. 2016;18(6):632-644.
57. Zhao S, et al. High expression of CD147 and MMP-9 is correlated with poor prognosis of triple-negative breast cancer (TNBC) patients. Med Oncol. 2013;30(1):335.

58. Heissig B, et al. Recruitment of stem and progenitor cells from the bone marrow niche requires MMP-9 mediated release of kit-ligand. Cell. 2002;109(5):625-637.

59. Visvader JE, Lindeman GJ. Cancer stem cells in solid tumours: accumulating evidence and unresolved questions. Nat Rev Cancer. 2008;8(10):755-768.

60. Thiagarajan PS, et al. Development of a fluorescent reporter system to delineate cancer stem cells in triple-negative breast cancer. Stem Cells. 2015;33(7):2114-2125.

61. Deome KB, Faulkin LJ Jr., Bern HA, Blair PB. Development of mammary tumors from hyperplastic alveolar nodules transplanted into glandfree mammary fat pads of female $\mathrm{C} 3 \mathrm{H}$ mice. Cancer Res. 1959;19(5):515-520.

62. Chakrabarti R, et al. Elf5 inhibits the epithelial-mesenchymal transition in mammary gland development and breast cancer metastasis by transcriptionally repressing Snail2. Nat Cell Biol. 2012;14(11):1212-1222.

63. Choi YS, Chakrabarti R, Escamilla-Hernandez R, Sinha S. Elf5 conditional knockout mice reveal its role as a master regulator in mammary alveolar development: failure of Stat5 activation and functional differentiation in the absence of Elf5. Dev Biol. 2009;329(2):227-241.

64. Sethi I, Gluck C, Zhou H, Buck M.J. Evolutionary re-wiring of $\mathrm{p} 63$ and the epigenomic regulatory landscape in keratinocytes and its potential implications on species-specific gene expression and phenotypes. Nucleic Acids Res. 2017; 45(14):8208-8224. 